$\mathrm{J}$ o u r n a l of

Mathematics

and Applications

JMA No 43, pp 19-45 (2020)

\title{
Relative Order and Relative Type Oriented Growth Properties of Generalized Iterated Entire Functions
}

\author{
Tanmay Biswas
}

\begin{abstract}
The main aim of this paper is to study some growth properties of generalized iterated entire functions in the light of their relative orders, relative types and relative weak types.
\end{abstract}

AMS Subject Classification: 30D20, 30D30, 30D35.

Keywords and Phrases: Entire function; Growth; Relative order; Relative type; Relative weak type; Composition; Property (A).

Let $\mathbb{C}$ be the set of all finite complex numbers. For any entire function $f(z)=$ $\sum_{n=0}^{\infty} a_{n} z^{n}$ defined in $\mathbb{C}$, the maximum modulus function $M_{f}(r)$ on $|z|=r$ is defined by $M_{f}(r)=\max _{|z|=r}|f(z)|$. If $f(z)$ is non-constant then $M_{f}(r)$ is strictly increasing and continuous. Also its inverse $M_{f}^{-1}:(|f(0)|, \infty) \rightarrow(0, \infty)$ exists and is such that $\lim _{s \rightarrow \infty} M_{f}^{-1}(s)=\infty$. Naturally, $M_{f}^{-1}(r)$ is also an increasing function of $r$. Also a non-constant entire function $f(z)$ is said to have the Property (A) if for any $\delta>1$ and for all sufficiently large $r,\left[M_{f}(r)\right]^{2} \leq M_{f}\left(r^{\delta}\right)$ holds (see [3]). For examples of functions with or without the Property (A), one may see [3]. In this connection Lahiri et al. (see [6]) prove that every entire function $f(z)$ satisfying the property (A) is transcendental. Moreover for any transcendental entire function $f(z)$, it is well known that $\lim _{r \rightarrow \infty} \frac{\log M_{f}(r)}{\log r}=\infty$ and for its application in growth measurement, one may see [8]. For another entire function $g(z)$, the ratio $\frac{M_{f}(r)}{M_{g}(r)}$ as $r \rightarrow \infty$ is called the growth of $f(z)$ with respect to $g(z)$ in terms of their maximum moduli. The notion of order and lower order which are the main tools to study the comparative growth properties of entire functions are very classical in complex analysis and their definitions are as follows:

COPYRIGHT (C) by Publishing House of Rzeszów University of Technology P.O. Box 85, 35-959 Rzeszów, Poland 
Definition 1. The order and the lower order of an entire function $f(z)$ denoted by $\rho(f)$ and $\lambda(f)$ respectively are defined as

$$
\begin{aligned}
& \rho(f) \\
& \lambda(f)
\end{aligned}=\lim _{r \rightarrow \infty} \sup _{\inf } \frac{\log \log M_{f}(r)}{\log \log M_{\exp z}(r)}=\lim _{r \rightarrow \infty} \sup _{\inf } \frac{\log \log M_{f}(r)}{\log r} .
$$

The rate of growth of an entire function generally depends upon order (respectively, lower order) of it. The entire function with higher order is of faster growth than that of lesser order. But if orders of two entire functions are same, then it is impossible to detect the function with faster growth. In that case, it is necessary to compute another class of growth indicators of entire functions called their types. Thus the type $\sigma(f)$ and lower type $\bar{\sigma}(f)$ of an entire function $f(z)$ are defined as:

Definition 2. Let $f(z)$ be an entire function with non zero finite order. Then the type $\sigma(f)$ and lower type $\bar{\sigma}(f)$ of an entire function $f(z)$ are defined as

$$
\begin{aligned}
& \sigma(f) \\
& \bar{\sigma}(f)
\end{aligned}=\lim _{r \rightarrow \infty} \sup _{\inf } \frac{\log M_{f}(r)}{\left(\log M_{\exp z}(r)\right)^{\rho(f)}}=\lim _{r \rightarrow \infty} \sup _{\inf } \frac{\log M_{f}(r)}{r^{\rho(f)}} .
$$

In order to calculate the order, it is seen that we have compared the maximum modulus of entire function $f(z)$ with that of $\exp z$ but here a question may arise why should we compare the maximum modulus of any entire function with that of only $\exp z$ whose growth rate is too high. From this view point, the relative order of entire functions may be thought of by Bernal (see $[2,3]$ ) who introduced the concept of relative order between two entire functions to avoid comparing growth just with $\exp z$. Thus the relative order of an entire function $f(z)$ with respect to an entire function $g(z)$, denoted by $\rho_{g}(f)$ is define as:

$$
\begin{aligned}
\rho_{g}(f) & =\inf \left\{\mu>0: M_{f}(r)<M_{g}\left(r^{\mu}\right) \text { for all } r>r_{0}(\mu)>0\right\} \\
& =\limsup _{r \rightarrow \infty} \frac{\log M_{g}^{-1}\left(M_{f}(r)\right)}{\log r} .
\end{aligned}
$$

Similarly, one can define the relative lower order of $f(z)$ with respect to $g(z)$ denoted by $\lambda_{g}(f)$ as follows :

$$
\lambda_{g}(f)=\liminf _{r \rightarrow \infty} \frac{\log M_{g}^{-1}\left(M_{f}(r)\right)}{\log r} .
$$

In the definition of relative order and relative lower order we generally compare the maximum modulus of any entire function $f(z)$ with that of any entire function $g(z)$ and it is quite natural that when $g(z)=\exp z$, both the definitions of relative order and relative lower order coincide with Definition 1.

In order to compare the relative growth of two entire functions having same non zero finite relative order with respect to another entire function, Roy [7] introduced the notion of relative type of two entire functions in the following way: 
Definition 3. [7] Let $f(z)$ and $g(z)$ be any two entire functions such that $0<\rho_{g}(f)<\infty$. Then the relative type $\sigma_{g}(f)$ of $f(z)$ with respect to $g(z)$ is defined as:

$$
\begin{aligned}
\sigma_{g}(f) & =\inf \left\{k>0: M_{f}(r)<M_{g}\left(k r^{\rho_{g}(f)}\right) \text { for all sufficiently large values of } r\right\} \\
& =\limsup _{r \rightarrow \infty} \frac{M_{g}^{-1}\left(M_{f}(r)\right)}{r^{\rho_{g}(f)}} .
\end{aligned}
$$

Similarly, one can define the relative lower type of an entire function $f(z)$ with respect to another entire function $g(z)$ denoted by $\bar{\sigma}_{g}(f)$ when $0<\rho_{g}(f)<\infty$ which is as follows:

$$
\bar{\sigma}_{g}(f)=\liminf _{r \rightarrow \infty} \frac{M_{g}^{-1}\left(M_{f}(r)\right)}{r^{\rho_{g}(f)}} .
$$

It is obvious that $0 \leq \bar{\sigma}_{g}(f) \leq \sigma_{g}(f) \leq \infty$.

If we consider $g(z)=\exp z$, then one can easily verify that Definition 3 coincides with the classical definitions of type and lower type respectively.

Like wise, to determine the relative growth of two entire functions having same non zero finite relative lower order with respect to another entire function, one may introduce the definition of relative weak type of an entire function $f(z)$ with respect to another entire function $g(z)$ of finite positive relative lower order $\lambda_{g}(f)$ in the following way:

Definition 4. Let $f(z)$ and $g(z)$ be any two entire functions such that $0<\lambda_{g}(f)<\infty$. The relative -weak type $\tau_{g}(f)$ and the growth indicator $\bar{\tau}_{g}(f)$ of an entire function $f(z)$ with respect to another entire function $g(z)$ are defined as:

$$
\begin{gathered}
\tau_{g}(f) \\
\bar{\tau}_{g}(f)
\end{gathered}=\lim _{r \rightarrow \infty} \inf \frac{M_{g}^{-1}\left(M_{f}(r)\right)}{r^{\lambda_{g}(f)}} .
$$

For any two entire functions $f(z), g(z)$ defined in $\mathbb{C}$ and for any real number $\alpha \in(0,1]$, Banerjee et al. [1] introduced the concept of generalized iteration of $f(z)$ with respect to $g(z)$ in the following manner:

$$
\begin{aligned}
& f_{1, g}(z)=(1-\alpha) z+\alpha f(z) \\
& f_{2, g}(z)=(1-\alpha) g_{1, f}(z)+\alpha f\left(g_{1, f}(z)\right) \\
& f_{3, g}(z)=(1-\alpha) g_{2, f}(z)+\alpha f\left(g_{2, f}(z)\right) \\
& \begin{array}{llll}
\ldots \ldots & \ldots \ldots & \ldots & \ldots
\end{array} \\
& \begin{array}{llll}
\ldots \ldots & \ldots \ldots & \ldots \ldots
\end{array} \\
& f_{n, g}(z)=(1-\alpha) g_{n-1, f}(z)+\alpha f\left(g_{n-1, f}(z)\right)
\end{aligned}
$$

and so

$$
\begin{aligned}
& g_{1, f}(z)=(1-\alpha) z+\alpha g(z) \\
& g_{2, f}(z)=(1-\alpha) f_{1, g}(z)+\alpha g\left(f_{1, g}(z)\right) \\
& g_{3, f}(z)=(1-\alpha) f_{2, g}(z)+\alpha g\left(f_{2, g}(z)\right) \\
& \text {.... } \ldots \ldots \quad \ldots \ldots \\
& \begin{array}{llll}
\ldots & \ldots \ldots & \ldots \ldots & \ldots \ldots
\end{array} \\
& g_{n, f}(z)=(1-\alpha) f_{n-1, g}(z)+\alpha g\left(f_{n-1, g}(z)\right) \text {. }
\end{aligned}
$$


Clearly all $f_{n, g}(z)$ and $g_{n, f}(z)$ are entire functions.

Further for another two non constant entire functions $h(z)$ and $k(z)$, one may define the iteration of $M_{h}{ }^{-1}(r)$ with respect to $M_{k}^{-1}(r)$ in the following manner:

$$
\begin{aligned}
& M_{h}^{-1}(r)=M_{h_{1}}^{-1}(r) \text {; } \\
& M_{k}^{-1}\left(M_{h}^{-1}(r)\right)=M_{k}^{-1}\left(M_{h_{1}}^{-1}(r)\right)=M_{h_{2}}^{-1}(r) \text {; } \\
& M_{h}^{-1}\left(M_{k}^{-1}\left(M_{h}^{-1}(r)\right)\right)=M_{h}^{-1}\left(M_{h_{2}}^{-1}(r)\right)=M_{h_{3}}^{-1}(r) \text {; } \\
& \text {.... } \quad \ldots \ldots, \quad \ldots \ldots \\
& \begin{array}{llll}
\ldots \ldots & \ldots \ldots & \ldots \ldots & \ldots \ldots
\end{array} \\
& M_{h}^{-1}\left(\ldots \ldots \ldots\left(M_{h}^{-1}\left(M_{k}^{-1}\left(M_{h}^{-1}(r)\right)\right)\right)\right)=M_{h_{n}}^{-1}(r) \text { when } n \text { is odd and } \\
& M_{k}^{-1}\left(\ldots \ldots \ldots\left(M_{h}^{-1}\left(M_{k}^{-1}\left(M_{h}^{-1}(r)\right)\right)\right)\right)=M_{h_{n}}^{-1}(r) \text { when } n \text { is even. }
\end{aligned}
$$

Obviously $M_{h_{n}}{ }^{-1}(r)$ is an increasing functions of $r$.

During the past decades, several researchers made close investigations on the growth properties of composite entire functions in different directions using their classical growth indicators such as order and type but the study of growth properties of composite entire functions using the concepts of relative order and relative type was mostly unknown to complex analysis which is and is the prime concern of the paper. The main aim of this paper is to study the growth properties of generalized iterated entire functions in almost a new direction in the light of their relative orders, relative types and relative weak types. Also our notation is standard within the theory of Nevanlinna's value distribution of entire functions which are available in [5] and [10]. Hence we do not explain those in details.

\section{Lemmas}

In this section we present some lemmas which will be needed in the sequel.

Lemma 1. [4] If $f(z)$ and $g(z)$ are any two entire functions with $g(0)=0$. Let $\beta$ satisfy $0<\beta<1$ and $c(\beta)=\frac{(1-\beta)^{2}}{4 \beta}$. Then for all sufficiently large values of $r$,

$$
M_{f}\left(c(\beta) M_{g}(\beta r)\right) \leq M_{f \circ g}(r) \leq M_{f}\left(M_{g}(r)\right)
$$

In addition if $\beta=\frac{1}{2}$, then for all sufficiently large values of $r$,

$$
M_{f \circ g}(r) \geq M_{f}\left(\frac{1}{8} M_{g}\left(\frac{r}{2}\right)\right) .
$$

Lemma 2. [3] Let $f(z)$ be an entire function which satisfies the Property (A). Then for any positive integer $n$ and for all large $r$,

$$
\left[M_{f}(r)\right]^{n} \leq M_{f}\left(r^{\delta}\right)
$$

holds where $\delta>1$. 
Lemma 3. [3] Let $f(z)$ be an entire function, $\alpha>1$ and $0<\beta<\alpha$. Then

$$
M_{f}(\alpha r)>\beta M_{f}(r)
$$

Lemma 4. Let $f(z), g(z)$ are any two transcendental entire functions and $h(z)$, $k(z)$ are any two entire functions such that $0<\rho_{h}(f)<\infty, 0<\rho_{k}(g)<\infty$ and $h(z), k(z)$ satisfy the Property (A). Then for all sufficiently large values of $r$,

$$
\text { (i) }\left(M_{h_{n}}^{-1}\left(M_{f_{n, g}}(r)\right)\right)^{\frac{1}{\delta}}<M_{k}^{-1}\left(M_{g}(r)\right) \text { when } n \text { is even }
$$

and

$$
\text { (ii) }\left(M_{h_{n}}^{-1}\left(M_{f_{n, g}}(r)\right)\right)^{\frac{1}{\delta}}<M_{h}^{-1}\left(M_{f}(r)\right) \text { when } n \text { is odd }
$$

where $\delta>1$.

Proof. Let $\beta$ be any positive integer such that $\max \left\{\rho_{h}(f), \rho_{k}(g)\right\}<\beta$ hold. Since for any transcendental entire function $f(z), \frac{\log M_{f}(r)}{\log r} \rightarrow \infty$ as $r \rightarrow \infty$, in view of Lemma 1, Lemma 2 and for any even integer $n$, we get for all sufficiently large values of $r$ that

$$
\begin{array}{ll} 
& M_{f_{n, g}}(r) \leq(1-\alpha) M_{g_{n-1, f}}(r)+\alpha M_{f\left(g_{n-1, f}\right)}(r) \\
\Rightarrow \quad & M_{f_{n, g}}(r)<(1-\alpha) M_{f}\left(M_{g_{n-1, f}}(r)\right)+\alpha M_{f}\left(M_{g_{n-1, f}}(r)\right) \\
\Rightarrow \quad & M_{h}^{-1}\left(M_{f_{n, g}}(r)\right)<M_{h}^{-1}\left(M_{f}\left(M_{g_{n-1, f}}(r)\right)\right) \\
\Rightarrow \quad & M_{h}^{-1}\left(M_{f_{n, g}}(r)\right)<\left(M_{g_{n-1, f}}(r)\right)^{\beta} \\
\Rightarrow \quad & \left(M_{h}^{-1}\left(M_{f_{n, g}}(r)\right)\right)^{\frac{1}{\beta}}<M_{g_{n-1, f}}(r) \\
\Rightarrow \quad & \left(M_{h}^{-1}\left(M_{f_{n, g}}(r)\right)\right)^{\frac{1}{\beta}}<(1-\alpha) M_{f_{n-2, g}}(r)+\alpha M_{g\left(f_{n-2, g}\right)}(r) \\
\Rightarrow \quad & \left(M_{h}^{-1}\left(M_{f_{n, g}}(r)\right)\right)^{\frac{1}{\beta}}<(1-\alpha) M_{g}\left(M_{f_{n-2, g}}(r)\right)+\alpha M_{g}\left(M_{f_{n-2, g}}(r)\right) \\
\Rightarrow \quad & M_{k}^{-1}\left(\left(M_{h}^{-1}\left(M_{f_{n, g}}(r)\right)\right)^{\frac{1}{\beta}}\right)<M_{k}^{-1}\left(M_{g}\left(M_{f_{n-2, g}}(r)\right)\right) \\
\Rightarrow \quad & \left(M_{k}^{-1}\left(M_{h}^{-1}\left(M_{f_{n, g}}(r)\right)\right)\right)^{\frac{1}{\delta}}<M_{k}^{-1}\left(M_{g}\left(M_{f_{n-2, g}}(r)\right)\right) \\
\Rightarrow \quad & \left(M_{k}^{-1}\left(M_{h}^{-1}\left(M_{f_{n, g}}(r)\right)\right)\right)^{\frac{1}{\delta}}<\left(M_{f_{n-2, g}}(r)\right)^{\beta} \\
\Rightarrow \quad & \left(M_{k}^{-1}\left(M_{h}^{-1}\left(M_{f_{n, g}}(r)\right)\right)\right)^{\frac{1}{\delta, \beta}}<M_{f_{n-2, g}}(r) \\
\Rightarrow \quad & \left(M_{h_{2}}^{-1}\left(M_{f_{n, g}}(r)\right)\right)^{\frac{1}{\delta, \beta}}<M_{f_{n-2, g}}(r) \\
\Rightarrow \quad & M_{h}^{-1}\left(\left(M_{h_{2}}^{-1}\left(M_{f_{n, g}}(r)\right)\right)^{\frac{1}{\delta, \beta}}<\left(M_{g_{n-3, f}}(r)\right)^{\beta}\right. \\
\Rightarrow \quad & \left(M_{h}^{-1}\left(M_{h_{2}}^{-1}\left(M_{f_{n, g}}(r)\right)\right)\right)^{\frac{1}{\delta}}<\left(M_{g_{n-3, f}}(r)\right)^{\beta} \\
\Rightarrow \quad & \left(M_{h_{3}}^{-1}\left(M_{f_{n, g}}(r)\right)\right)^{\frac{1}{\delta, \beta}}<M_{g_{n-3, f}}(r)
\end{array}
$$




$$
\begin{aligned}
& \Rightarrow \quad M_{k}^{-1}\left(\left(M_{h_{3}}^{-1}\left(M_{f_{n, g}}(r)\right)\right)^{\frac{1}{\delta \cdot \beta}}\right)<\left(M_{f_{n-4, g}}(r)\right)^{\beta} \\
& \Rightarrow \quad\left(M_{h_{4}}^{-1}\left(M_{f_{n, g}}(r)\right)\right)^{\frac{1}{\delta, \beta}}<M_{f_{n-4, g}}(r)
\end{aligned}
$$

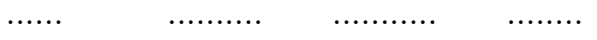

Therefore

$$
\left(M_{h_{n}}^{-1}\left(M_{f_{n, g}}(r)\right)\right)^{\frac{1}{\delta}}<M_{k}^{-1}\left(M_{g}(r)\right) \text { when } n \text { is even. }
$$

Similarly,

$$
\left(M_{h_{n}}^{-1}\left(M_{f_{n, g}}(r)\right)\right)^{\frac{1}{\delta}}<M_{h}^{-1}\left(M_{f}(r)\right) \text { when } n \text { is odd } .
$$

Hence the lemma follows.

Remark 1. If we consider $0<\rho_{h}(f) \leq 1$ and $0<\rho_{k}(g) \leq 1$ in Lemma 4 , then it is not necessary for both $h(z)$ and $k(z)$ to satisfy Property $(\mathrm{A})$ and in this case Lemma 4 holds with $\delta=1$.

Lemma 5. Let $f(z), g(z)$ are any two transcendental entire functions and $h(z)$, $k(z)$ are any two entire functions such that $0<\lambda_{h}(f)<\infty, 0<\lambda_{k}(g)<\infty$ and $h(z), k(z)$ satisfy the Property (A). Also let $\delta>1,0<\beta<\alpha<1$, $\omega$ is a positive integer such that $\min \left\{\lambda_{h}(f), \lambda_{k}(g)\right\}>\frac{1}{\omega}$ and $\gamma_{n}>\frac{\gamma_{n-1}^{\omega}}{(\alpha-\beta)}$ where $\gamma_{0}=1$. Then for all sufficiently large values of $r$,

(i) $\gamma_{n}\left(M_{h_{n}}^{-1}\left(M_{f_{n, g}}(r)\right)\right)^{\delta}>M_{k}^{-1}\left(M_{g}\left(\frac{r}{18^{n}}\right)\right)$ when $n$ is even

and

(ii) $\gamma_{n}\left(M_{h_{n}}^{-1}\left(M_{f_{n, g}}(r)\right)\right)^{\delta}>M_{h}^{-1}\left(M_{f}\left(\frac{r}{18^{n}}\right)\right)$ when $n$ is odd.

Proof. Since for any transcendental entire function $f, \frac{\log M_{f}(r)}{\log r} \rightarrow \infty$ as $r \rightarrow \infty$, therefore $\frac{\log \frac{\beta}{(1-\alpha)} M_{f}(r)}{\log r} \rightarrow \infty$ as $r \rightarrow \infty$ where $0<\beta<\alpha$. Hence in view of Lemma 1 , Lemma 2, Lemma 3 and for any even integer $n$, we get for all sufficiently large values of $r$ that

$$
M_{f_{n, g}}(r) \geq \alpha M_{f\left(g_{n-1, f}\right)}(r)-(1-\alpha) M_{g_{n-1, f}}(r)
$$




$$
\begin{aligned}
& \Rightarrow \quad M_{f_{n, g}}(r)>\alpha M_{f}\left(M_{g_{n-1, f}}\left(\frac{r}{18}\right)\right)-\beta M_{f}\left(M_{g_{n-1, f}}\left(\frac{r}{18}\right)\right) \\
& \Rightarrow \quad M_{f_{n, g}}(r)>(\alpha-\beta) M_{f}\left(M_{g_{n-1, f}}\left(\frac{r}{18}\right)\right) \\
& \Rightarrow \quad M_{h}^{-1}\left(\frac{1}{(\alpha-\beta)} M_{f_{n, g}}(r)\right)>M_{h}^{-1}\left(M_{f}\left(M_{g_{n-1, f}}\left(\frac{r}{18}\right)\right)\right) \\
& \Rightarrow \quad M_{h}^{-1}\left(\frac{1}{(\alpha-\beta)} M_{f_{n, g}}(r)\right)>\left(M_{g_{n-1, f}}\left(\frac{r}{18}\right)\right)^{\frac{1}{\omega}} \\
& \Rightarrow \quad\left(\gamma_{1} M_{h}^{-1}\left(M_{f_{n, g}}(r)\right)\right)^{\omega}>M_{g_{n-1, f}}\left(\frac{r}{18}\right) \\
& \Rightarrow \quad \gamma_{1}^{\omega}\left(M_{h}^{-1}\left(M_{f_{n, g}}(r)\right)\right)^{\omega}>\alpha M_{g}\left(M_{f_{n-2, g}}\left(\frac{r}{18^{2}}\right)\right)-\beta M_{g}\left(M_{f_{n-2, g}}\left(\frac{r}{18^{2}}\right)\right) \\
& \Rightarrow \quad \gamma_{1}^{\omega}\left(M_{h}^{-1}\left(M_{f_{n, g}}(r)\right)\right)^{\omega}>(\alpha-\beta) M_{g}\left(M_{f_{n-2, g}}\left(\frac{r}{18^{2}}\right)\right) \\
& \Rightarrow \quad \frac{\gamma_{1}^{\omega}}{(\alpha-\beta)}\left(M_{h}^{-1}\left(M_{f_{n, g}}(r)\right)\right)^{\omega}>M_{g}\left(M_{f_{n-2, g}}\left(\frac{r}{18^{2}}\right)\right) \\
& \Rightarrow \quad M_{k}^{-1}\left(\frac{\gamma_{1}^{\omega}}{(\alpha-\beta)}\left(M_{h}^{-1}\left(M_{f_{n, g}}(r)\right)\right)^{\omega}\right)>M_{k}^{-1}\left(M_{g}\left(M_{f_{n-2, g}}\left(\frac{r}{18^{2}}\right)\right)\right) \\
& \Rightarrow \quad \gamma_{2}\left(M_{k}^{-1}\left(M_{h}^{-1}\left(M_{f_{n, g}}(r)\right)\right)\right)^{\delta}>\left(M_{f_{n-2, g}}\left(\frac{r}{18^{2}}\right)\right)^{\frac{1}{\omega}} \\
& \Rightarrow \quad \gamma_{2}^{\omega}\left(M_{h_{2}}^{-1}\left(M_{f_{n, g}}(r)\right)\right)^{\delta \omega}>M_{f_{n-2, g}}\left(\frac{r}{18^{2}}\right) \\
& \Rightarrow \quad M_{h}^{-1}\left(\frac{\gamma_{2}^{\omega}}{(\alpha-\beta)}\left(M_{h_{2}}^{-1}\left(M_{f_{n, g}}(r)\right)\right)^{\delta \omega}\right)>\left(M_{g_{n-3, f}}\left(\frac{r}{18^{3}}\right)\right)^{\frac{1}{\omega}} \\
& \Rightarrow \quad \gamma_{3}^{\omega}\left(M_{h}^{-1}\left(M_{h_{2}}^{-1}\left(M_{f_{n, g}}(r)\right)\right)\right)^{\delta \omega}>M_{g_{n-3, f}}\left(\frac{r}{18^{3}}\right) \\
& \Rightarrow \quad \gamma_{3}^{\omega}\left(M_{h_{3}}^{-1}\left(M_{f_{n, g}}(r)\right)\right)^{\delta \omega}>M_{g_{n-3, f}}\left(\frac{r}{18^{3}}\right) \\
& \Rightarrow \quad \gamma_{4}^{\omega}\left(M_{k}^{-1}\left(M_{h_{3}}^{-1}\left(M_{f_{n, g}}(r)\right)\right)\right)^{\delta \omega}>M_{f_{n-4, g}}\left(\frac{r}{18^{4}}\right) \\
& \Rightarrow \quad \gamma_{4}^{\omega}\left(M_{h_{4}}^{-1}\left(M_{f_{n, g}}(r)\right)\right)^{\delta \omega}>M_{f_{n-4, g}}\left(\frac{r}{18^{4}}\right) \\
& \cdots \cdots
\end{aligned}
$$

Therefore

$$
\gamma_{n}\left(M_{h_{n}}^{-1}\left(M_{f_{n, g}}(r)\right)\right)^{\delta}>M_{k}^{-1}\left(M_{g}\left(\frac{r}{18^{n}}\right)\right) \text { when } n \text { is even. }
$$

Similarly,

$$
\gamma_{n}\left(M_{h_{n}}^{-1}\left(M_{f_{n, g}}(r)\right)\right)^{\delta}>M_{h}^{-1}\left(M_{f}\left(\frac{r}{18^{n}}\right)\right) \text { when } n \text { is odd. }
$$

Hence the lemma follows.

Remark 2. If we consider $1 \leq \lambda_{h}(f)<\infty$ and $1 \leq \lambda_{k}(g)<\infty$ in Lemma 5 , then it 
is not necessary for both $h$ and $k$ to satisfy Property (A) and in this case Lemma 5 holds with $\delta=1$.

\section{Main Results}

In this section we present the main results of the paper. Throughout the paper, we consider the entire functions $H(z), K(z), h(z), k(z)$ satisfy the Property (A) as and when necessary. Also consider that $F(z), G(z), f(z), g(z)$ are non constant entire functions.

Theorem 1. Let $f(z), g(z), k(z)$ and $h(z)$ be any four entire functions such that $0<\lambda_{h}(f) \leq \rho_{h}(f)<\infty, 0<\lambda_{k}(g)<\infty$ and $0<\mu<\rho_{k}(g)<\infty$. Then for any even number $n$,

$$
\limsup _{r \rightarrow \infty} \frac{M_{h_{n}}^{-1}\left(M_{f_{n, g}}(r)\right)}{\log M_{h}^{-1} M_{f}\left(\exp r^{\delta \mu}\right)}=\infty
$$

where $\delta<1$.

Proof. From the first part of Lemma 5, we get for a sequence of values of $r$ tending to infinity that

$$
M_{h_{n}}^{-1}\left(M_{f_{n, g}}(r)\right)>\left(\frac{1}{\gamma_{n}}\right)^{\delta}\left(\frac{r}{18^{n}}\right)^{\delta\left(\rho_{k}(g)-\varepsilon\right)},
$$

where $\gamma_{n}$ is defined in Lemma 5 .

Again from the definition of $\rho_{h}(f)$, we obtain for all sufficiently large values of $r$ that

$$
\log M_{h}^{-1}\left(M_{f}\left(\exp r^{\delta \mu}\right)\right) \leq\left(\rho_{h}(f)+\varepsilon\right) r^{\delta \mu}
$$

Now from (2.1) and (2.2), it follows for a sequence of values of $r$ tending to infinity that

$$
\frac{M_{h_{n}}^{-1}\left(M_{f_{n, g}}(r)\right)}{\log M_{h}^{-1}\left(M_{f}\left(\exp r^{\delta \mu}\right)\right)}>\frac{\left(\frac{1}{\gamma_{n}}\right)^{\delta}\left(\frac{r}{18^{n}}\right)^{\delta\left(\rho_{k}(g)-\varepsilon\right)}}{\left(\rho_{h}(f)+\varepsilon\right) r^{\delta \mu}} .
$$

As $\mu<\rho_{k}(g)$, we can choose $\varepsilon(>0)$ in such a way that

$$
\mu<\rho_{k}(g)-\varepsilon
$$

Thus from (2.3) and (2.4) we get that

$$
\limsup _{r \rightarrow \infty} \frac{M_{h_{n}}^{-1}\left(M_{f_{n, g}}(r)\right)}{\log M_{h}^{-1}\left(M_{f}\left(\exp r^{\delta \mu}\right)\right)}=\infty .
$$

Hence the theorem follows from (2.5). 
Theorem 2. Let $f(z), g(z), k(z)$ and $h(z)$ be any four entire functions such that $0<\lambda_{h}(f) \leq \rho_{h}(f)<\infty, 0<\lambda_{k}(g)<\infty$ and $0<\mu<\rho_{k}(g)<\infty$. Then for any even number $n$,

$$
\limsup _{r \rightarrow \infty} \frac{M_{h_{n}}^{-1}\left(M_{f_{n, g}}(r)\right)}{\log M_{k}^{-1}\left(M_{g}\left(\exp r^{\delta \mu}\right)\right)}=\infty,
$$

where $\delta<1$.

Proof. Let $0<\mu<\mu_{0}<\rho_{k}(g)$. Then from (2.5), we obtain for a sequence of values of $r$ tending to infinity and $A>1$ that

$$
\begin{aligned}
M_{h_{n}}^{-1}\left(M_{f_{n, g}}(r)\right) & >A \log M_{h}^{-1}\left(M_{f}\left(\exp r^{\delta \mu_{0}}\right)\right) \\
\text { i.e., } M_{h_{n}}^{-1}\left(M_{f_{n, g}}(r)\right) & >A\left(\lambda_{h}(f)-\varepsilon\right) r^{\delta \mu_{0}} .
\end{aligned}
$$

Again from the definition of $\rho_{k}(g)$, we obtain for all sufficiently large values of $r$ that

$$
\log M_{k}^{-1}\left(M_{g}\left(\exp r^{\delta \mu}\right)\right) \leq\left(\rho_{k}(g)+\varepsilon\right) r^{\delta \mu} .
$$

So combining (2.6) and (2.7), we obtain for a sequence of values of $r$ tending to infinity that

$$
\frac{M_{h_{n}}^{-1}\left(M_{f_{n, g}}(r)\right)}{\log M_{k}^{-1}\left(M_{g}\left(\exp r^{\delta \mu}\right)\right)}>\frac{A\left(\lambda_{h}(f)-\varepsilon\right) r^{\delta \mu_{0}}}{\left(\rho_{k}(g)+\varepsilon\right) r^{\delta \mu}} .
$$

Since $\mu_{0}>\mu$, from (2.8) it follows that

$$
\limsup _{r \rightarrow \infty} \frac{M_{h_{n}}^{-1}\left(M_{f_{n, g}}(r)\right)}{\log M_{k}^{-1}\left(M_{g}\left(\exp r^{\delta \mu}\right)\right)}=\infty .
$$

Thus the theorem follows.

Now we state the following two theorems without their proofs as those can easily be carried out in the line of Theorem 1 and Theorem 2 respectively and with the help of the second part of Lemma 5 .

Theorem 3. Let $f(z), g(z), k(z)$ and $h(z)$ be any four entire functions such that $0<\lambda_{k}(g) \leq \rho_{k}(g)<\infty, 0<\lambda_{h}(f)<\infty$ and $0<\mu<\rho_{h}(f)<\infty$. Then for any odd number n,

$$
\limsup _{r \rightarrow \infty} \frac{M_{h_{n}}^{-1}\left(M_{f_{n, g}}(r)\right)}{\log M_{h}^{-1}\left(M_{f}\left(\exp r^{\delta \mu}\right)\right)}=\infty,
$$

where $\delta<1$.

Theorem 4. Let $f(z), g(z), k(z)$ and $h(z)$ be any four entire functions such that $0<\lambda_{k}(g) \leq \rho_{k}(g)<\infty, 0<\lambda_{h}(f)<\infty$ and $0<\mu<\rho_{h}(f)<\infty$. Then for any odd number $n$,

$$
\limsup _{r \rightarrow \infty} \frac{M_{h_{n}}^{-1}\left(M_{f_{n, g}}(r)\right)}{\log M_{k}^{-1}\left(M_{g}\left(\exp r^{\delta \mu}\right)\right)}=\infty,
$$

where $\delta<1$. 
Theorem 5. Let $f(z), g(z), k(z)$ and $h(z)$ be any four entire functions such that $0<\lambda_{h}(f) \leq \rho_{h}(f)<\infty, 0<\rho_{k}(g)<\infty$ and $\lambda_{k}(g)<\mu<\infty$. Then for any even number $n$,

$$
\liminf _{r \rightarrow \infty} \frac{M_{h_{n}}^{-1}\left(M_{f_{n, g}}(r)\right)}{\log M_{h}^{-1}\left(M_{f}\left(\exp r^{\delta \mu}\right)\right)}=0
$$

where $\delta>1$.

Proof. From the first part of Lemma 4, it follows for a sequence of values of $r$ tending to infinity that

$$
M_{h_{n}}^{-1}\left(M_{f_{n, g}}(r)\right)<r^{\delta\left(\lambda_{k}(g)+\varepsilon\right)} .
$$

Again for all sufficiently large values of $r$ we get that

$$
\log M_{h}^{-1}\left(M_{f}\left(\exp r^{\delta \mu}\right)\right) \geq\left(\lambda_{h}(f)-\varepsilon\right) r^{\delta \mu} .
$$

Now from (2.9) and (2.10), it follows for a sequence of values of $r$ tending to infinity that

$$
\frac{M_{h_{n}}^{-1}\left(M_{f_{n, g}}(r)\right)}{\log M_{h}^{-1}\left(M_{f}\left(\exp r^{\delta \mu}\right)\right)}<\frac{r^{\delta\left(\lambda_{k}(g)+\varepsilon\right)}}{\left(\lambda_{h}(f)-\varepsilon\right) r^{\delta \mu}} .
$$

As $\lambda_{k}(g)<\mu$, we can choose $\varepsilon(>0)$ in such a way that

$$
\lambda_{k}(g)+\varepsilon<\mu .
$$

Thus the theorem follows from (2.11) and (2.12).

In the line of Theorem 5, we may state the following theorem without its proof:

Theorem 6. Let $f(z), g(z), k(z)$ and $h(z)$ be any four entire functions such that $0<\rho_{h}(f)<\infty, 0<\rho_{k}(g)<\infty$ and $\lambda_{k}(g)<\mu<\infty$. Then for any even number $n$,

$$
\liminf _{r \rightarrow \infty} \frac{M_{h_{n}}^{-1}\left(M_{f_{n, g}}(r)\right)}{\log M_{k}^{-1}\left(M_{g}\left(\exp r^{\delta \mu}\right)\right)}=0
$$

where $\delta>1$.

Theorem 7. Let $f(z), g(z), k(z)$ and $h(z)$ be any four entire functions such that $0<\lambda_{k}(g) \leq \rho_{k}(g)<\infty, 0<\rho_{h}(f)<\infty$ and $\lambda_{h}(f)<\mu<\infty$. Then for any odd number $n$,

where $\delta>1$.

$$
\liminf _{r \rightarrow \infty} \frac{M_{h_{n}}^{-1}\left(M_{f_{n, g}}(r)\right)}{\log M_{k}^{-1}\left(M_{g}\left(\exp r^{\delta \mu}\right)\right)}=0
$$

Theorem 8. Let $f(z), g(z), k(z)$ and $h(z)$ be any four entire functions such that Let $f(z), g(z), k(z)$ and $h(z)$ be any four entire functions such that $0<\rho_{k}(g)<\infty$, $0<\rho_{h}(f)<\infty$ and $\lambda_{h}(f)<\mu<\infty$. Then for any odd number $n$,

$$
\liminf _{r \rightarrow \infty} \frac{M_{h_{n}}^{-1}\left(M_{f_{n, g}}(r)\right)}{\log M_{h}^{-1}\left(M_{f}\left(\exp r^{\delta \mu}\right)\right)}=0
$$

where $\delta>1$. 
We omit the proofs of Theorem 7 and Theorem 8 as those can be carried out in the line of Theorem 5 and Theorem 6 respectively and with the help of the second part of Lemma 4.

Theorem 9. Let $f(z), g(z), k(z)$ and $h(z)$ be any four entire functions such that $0<\lambda_{h}(f) \leq \rho_{h}(f)<\infty$ and $0<\lambda_{k}(g)<\infty$. Also let $\gamma$ be a positive continuous on $[0,+\infty)$ function increasing to $+\infty$. Then for every real number $\kappa$ and positive integer $n$

$$
\lim _{r \rightarrow \infty} \frac{M_{h_{n}}^{-1}\left(M_{f_{n, g}}(r)\right)}{\left\{\log M_{h}^{-1}\left(M_{f}(\exp \gamma(r))\right)\right\}^{1+\kappa}}=\infty,
$$

where

$$
\lim _{r \rightarrow \infty} \frac{\log \gamma(r)}{\log r}=0
$$

Proof. First let us consider $n$ to be an even integer. If $\kappa$ be such that $1+\kappa \leq 0$ then the theorem is trivial. So we suppose that $1+\kappa>0$. Now it follows from the first part of Lemma 5, for all sufficiently large values of $r$ that

$$
M_{h_{n}}^{-1}\left(M_{f_{n, g}}(r)\right)>\left(\frac{1}{\gamma_{n}}\right)^{\frac{1}{\delta}}\left(\frac{r}{18^{n}}\right)^{\frac{\lambda_{k}(g)-\varepsilon}{\delta}}
$$

where $\delta$ and $\gamma_{n}$ are defined in Lemma 5 . that

Again from the definition of $\rho_{h}(f)$, it follows for all sufficiently large values of $r$

$$
\left\{\log M_{h}^{-1}\left(M_{f}(\exp \gamma(r))\right\}^{1+\kappa} \leq\left(\rho_{h}(f)+\varepsilon\right)^{1+\kappa}(\gamma(r))^{1+\kappa}\right.
$$

Now from (2.13) and (2.14), it follows for all sufficiently large values of $r$ that

$$
\frac{M_{h_{n}}^{-1}\left(M_{f_{n, g}}(r)\right)}{\left\{\log M_{h}^{-1}\left(M_{f}(\exp \gamma(r))\right)\right\}^{1+\kappa}}>\frac{\left(\frac{1}{\gamma_{n}}\right)^{\frac{1}{\delta}} \cdot\left(\frac{1}{18^{n}}\right)^{\frac{\lambda_{k}(g)-\varepsilon}{\delta}} \cdot r^{\frac{\lambda_{k}(g)-\varepsilon}{\delta}}}{\left(\rho_{h}(f)+\varepsilon\right)^{1+\kappa}(\gamma(r))^{1+\kappa}} .
$$

Since $\lim _{r \rightarrow \infty} \frac{\log \gamma(r)}{\log r}=0$, therefore $\frac{r^{\frac{\lambda_{k}(g)-\varepsilon}{\delta}}}{(\gamma(r))^{1+\kappa}} \rightarrow \infty$ as $r \rightarrow \infty$, then from above it follows that

$$
\liminf _{r \rightarrow \infty} \frac{M_{h_{n}}^{-1}\left(M_{f_{n, g}}(r)\right)}{\left\{\log M_{h}^{-1}\left(M_{f}(\exp \gamma(r))\right)\right\}^{1+\kappa}}=\infty \text { for any even number } n .
$$

Similarly, with the help of the second part of Lemma 5 one can easily derive the same conclusion for any odd integer $n$.

Hence the theorem follows.

Remark 3. Theorem 9 is still valid with "limit superior" instead of "limit" if we replace the condition " $0<\lambda_{h}(f) \leq \rho_{h}(f)<\infty$ " by " $0<\lambda_{h}(f)<\infty$ ".

In the line of Theorem 9, one may state the following theorem without its proof: 
Theorem 10. Let $f(z), g(z), k(z)$ and $h(z)$ be any four entire functions such that $0<\lambda_{h}(f)<\infty$ and $0<\lambda_{k}(g) \leq \rho_{k}(g)<\infty$. Also let $\gamma$ be a positive continuous on $[0,+\infty)$ function increasing to $+\infty$. Then for every real number $\kappa$ and positive integer $n$

$$
\lim _{r \rightarrow \infty} \frac{M_{h_{n}}^{-1}\left(M_{f_{n, g}}(r)\right)}{\left\{\log M_{k}^{-1}\left(M_{g}(\exp \gamma(r))\right)\right\}^{1+\kappa}}=\infty,
$$

where

$$
\lim _{r \rightarrow \infty} \frac{\log \gamma(r)}{\log r}=0
$$

Remark 4. In Theorem 10 if we take the condition $0<\lambda_{k}(g)<\infty$ instead of $0<\lambda_{k}(g) \leq \rho_{k}(g)<\infty$, then also Theorem 10 remains true with "limit superior" in place of "limit".

Theorem 11. Let $f(z), g(z), k(z)$ and $h(z)$ be any four entire functions such that $0<\lambda_{h}(f) \leq \rho_{h}(f)<\infty$ and $0<\rho_{k}(g)<\infty$. Also let $\gamma$ be a positive continuous on $[0,+\infty)$ function increasing to $+\infty$. Then for each $\kappa \in(-\infty, \infty)$ and positive integer $n$

$$
\lim _{r \rightarrow \infty} \frac{\left(M_{h_{n}}^{-1}\left(M_{f_{n, g}}(r)\right)\right)^{1+\kappa}}{\log M_{h}^{-1}\left(M_{f}(\exp \gamma(r))\right)}=0
$$

where

$$
\lim _{r \rightarrow \infty} \frac{\log \gamma(r)}{\log r}=\infty
$$

Proof. If $1+\kappa \leq 0$, then the theorem is obvious. We consider that $1+\kappa>0$. Also let us consider $n$ to be an even integer. Now it follows from the first part of Lemma 4 for all sufficiently large values of $r$ that

$$
M_{h_{n}}^{-1}\left(M_{f_{n, g}}(r)\right)<r^{\delta\left(\rho_{k}(g)+\varepsilon\right)},
$$

where $\delta>1$.

Again for all sufficiently large values of $r$ we get that

$$
\log M_{h}^{-1}\left(M_{f}(\exp \gamma(r))\right) \geq\left(\lambda_{h}(f)-\varepsilon\right) \gamma(r) .
$$

Hence for all sufficiently large values of $r$, we obtain from (2.15) and (2.16) that

$$
\frac{\left(M_{h_{n}}^{-1}\left(M_{f_{n, g}}(r)\right)\right)^{1+\kappa}}{\log M_{h}^{-1}\left(M_{f}(\exp \gamma(r))\right)}<\frac{r^{\delta\left(\rho_{k}(g)+\varepsilon\right)(1+\kappa)}}{\left(\lambda_{h}(f)-\varepsilon\right) \gamma(r)},
$$

where we choose $0<\varepsilon<\min \left\{\lambda_{h}(f), \rho_{k}(g)\right\}$.

Since $\lim _{r \rightarrow \infty} \frac{\log \gamma(r)}{\log r}=\infty$, therefore $\frac{r^{\delta\left(\rho_{k}(g)+\varepsilon\right)(1+\kappa)}}{\gamma(r)} \rightarrow \infty$ as $r \rightarrow \infty$, then from $(2.17)$ we obtain that

$$
\liminf _{r \rightarrow \infty} \frac{\left(M_{h_{n}}^{-1}\left(M_{f_{n, g}}(r)\right)\right)^{1+\kappa}}{\log M_{h}^{-1}\left(M_{f}(\exp \gamma(r))\right)}=0 \text { for any even number } n .
$$


Similarly, with the help of the second part of Lemma 4 one can easily derive the same conclusion for any odd integer $n$.

This proves the theorem.

Remark 5. In Theorem 11 if we take the condition $0<\rho_{h}(f)<\infty$ instead of $0<\lambda_{h}(f) \leq \rho_{h}(f)<\infty$, the theorem remains true with "limit inferior" in place of "limit".

In view of Theorem 11, the following theorem can be carried out :

Theorem 12. Let $f(z), g(z), k(z)$ and $h(z)$ be any four entire functions such that $0<\rho_{h}(f)<\infty$ and $0<\lambda_{k}(g) \leq \rho_{k}(g)<\infty$. Also let $\gamma$ be a positive continuous on $[0,+\infty)$ function increasing to $+\infty$. Then for each $\kappa \in(-\infty, \infty)$ and positive integer $n$

$$
\lim _{r \rightarrow \infty} \frac{\left(M_{h_{n}}^{-1}\left(M_{f_{n, g}}(r)\right)\right)^{1+\kappa}}{\log M_{k}^{-1}\left(M_{g}(\exp \gamma(r))\right)}=0,
$$

where

$$
\lim _{r \rightarrow \infty} \frac{\log \gamma(r)}{\log r}=\infty
$$

The proof is omitted.

Remark 6. In Theorem 12 if we take the condition $0<\rho_{k}(g)<\infty$ instead of $0<\lambda_{k}(g) \leq \rho_{k}(g)<\infty$ then the theorem remains true with "limit inferior" in place of "limit".

Theorem 13. Let $f(z), g(z), k(z)$ and $h(z)$ be any four entire functions such that $\lambda_{k}(g)<\lambda_{h}(f) \leq \rho_{h}(f)<\infty$ and $0<\rho_{k}(g)<\infty$. Then for any even number $n$,

$$
\liminf _{r \rightarrow \infty} \frac{M_{h_{n}}^{-1}\left(M_{f_{n, g}}(r)\right)}{M_{h}^{-1}\left(M_{f}\left(r^{\delta}\right)\right)}=0,
$$

where $\delta>1$.

Proof. From the first part of Lemma 4, we obtain for a sequence of values of $r$ tending to infinity that

$$
M_{h_{n}}^{-1}\left(M_{f_{n, g}}(r)\right)<r^{\delta\left(\lambda_{k}(g)+\varepsilon\right)} .
$$

Again from the definition of relative order, we obtain for all sufficiently large values of $r$ that

$$
M_{h}^{-1}\left(M_{f}\left(r^{\delta}\right)\right) \geqslant r^{\delta\left(\lambda_{h}(f)-\varepsilon\right)} .
$$

Now in view of (2.18) and (2.19), we get for a sequence of values of $r$ tending to infinity that

$$
\frac{M_{h_{n}}^{-1}\left(M_{f_{n, g}}(r)\right)}{M_{h}^{-1}\left(M_{f}\left(r^{\delta}\right)\right)}<\frac{r^{\delta\left(\lambda_{k}(g)+\varepsilon\right)}}{r^{\delta\left(\lambda_{h}(f)-\varepsilon\right)}} .
$$

Since $\lambda_{k}(g)<\lambda_{h}(f)$, we can choose $\varepsilon(>0)$ in such a way that $\lambda_{k}(g)+\varepsilon<\lambda_{h}(f)-\varepsilon$ and then the theorem follows from $(2.20)$. 
Remark 7. If we take $0<\rho_{k}(g)<\lambda_{h}(f) \leq \rho_{h}(f)<\infty$ instead of " $\lambda_{k}(g)<$ $\lambda_{h}(f) \leq \rho_{h}(f)<\infty$ and $\rho_{k}(g)<\infty$ " and the other conditions remain the same, the conclusion of Theorem 13 remains valid with "limit inferior" replaced by "limit".

Theorem 14. Let $f(z), g(z), k(z)$ and $h(z)$ be any four entire functions such that $\lambda_{h}(f)<\lambda_{k}(g) \leq \rho_{k}(g)<\infty$ and $0<\rho_{h}(f)<\infty$. Then for any odd number $n$,

$$
\liminf _{r \rightarrow \infty} \frac{M_{h_{n}}^{-1}\left(M_{f_{n, g}}(r)\right)}{M_{k}^{-1}\left(M_{g}\left(r^{\delta}\right)\right)}=0
$$

where $\delta>1$.

The proof of Theorem 14 is omitted as it can be carried out in the line of Theorem 13 and with the help of the second part of Lemma 4.

Remark 8. If we consider $0<\rho_{h}(f)<\lambda_{k}(g) \leq \rho_{k}(g)<\infty$ instead of " $\lambda_{h}(f)<$ $\lambda_{k}(g) \leq \rho_{k}(g)<\infty$ and $\rho_{h}(f)<\infty$ " and the other conditions remain the same, the conclusion of Theorem 13 remains valid with "limit inferior" replaced by "limit".

Theorem 15. Let $f(z), g(z), k(z)$ and $h(z)$ be any four entire functions such that $0<\lambda_{h}(f) \leq \rho_{h}(f)<\infty$ and $0<\rho_{k}(g)<\infty$. Then

$$
\limsup _{r \rightarrow \infty} \frac{\log M_{h_{n}}^{-1}\left(M_{f_{n, g}}(r)\right)}{\log M_{h}^{-1}\left(M_{f}\left(r^{\delta}\right)\right)} \leq \frac{\rho_{k}(g)}{\lambda_{h}(f)} \text { when } n \text { is even }
$$

and

$$
\limsup _{r \rightarrow \infty} \frac{\log M_{h_{n}}^{-1}\left(M_{f_{n, g}}(r)\right)}{\log M_{h}^{-1}\left(M_{f}\left(r^{\delta}\right)\right)} \leq \frac{\rho_{h}(f)}{\lambda_{h}(f)} \text { when } n \text { is any odd integer }
$$

where $\delta>1$.

Proof. From the first part of Lemma 4, it follows for all sufficiently large values of $r$ that

$$
\begin{aligned}
& \frac{\log M_{h_{n}}^{-1}\left(M_{f_{n, g}}(r)\right)}{\log M_{h}^{-1}\left(M_{f}\left(r^{\delta}\right)\right)}<\frac{\delta \log M_{k}^{-1}\left(M_{g}(r)\right)}{\log M_{h}^{-1}\left(M_{f}\left(r^{\delta}\right)\right)} \\
& \text { i.e., } \frac{\log M_{h_{n}}^{-1}\left(M_{f_{n, g}}(r)\right)}{\log M_{h}^{-1}\left(M_{f}\left(r^{\delta}\right)\right)}<\frac{\delta \log M_{k}^{-1}\left(M_{g}(r)\right)}{\delta \log r} \cdot \frac{\log r^{\delta}}{\log M_{h}^{-1}\left(M_{f}\left(r^{\delta}\right)\right)}
\end{aligned}
$$

i.e., $\limsup _{r \rightarrow \infty} \frac{\log M_{h_{n}}^{-1}\left(M_{f_{n, g}}(r)\right)}{\log M_{h}^{-1}\left(M_{f}\left(r^{\delta}\right)\right)} \leq \limsup _{r \rightarrow \infty} \frac{\log M_{k}^{-1}\left(M_{g}(r)\right)}{\log r} \cdot \limsup _{r \rightarrow \infty} \frac{\log r^{\delta}}{\log M_{h}^{-1}\left(M_{f}\left(r^{\delta}\right)\right)}$

i.e., $\limsup _{r \rightarrow \infty} \frac{\log M_{h_{n}}^{-1}\left(M_{f_{n, g}}(r)\right)}{\log M_{h}^{-1}\left(M_{f}\left(r^{\delta}\right)\right)} \leq \rho_{k}(g) \cdot \frac{1}{\lambda_{h}(f)}=\frac{\rho_{k}(g)}{\lambda_{h}(f)}$.

Thus the first part of theorem follows from above.

Similarly, with the help of the second part of Lemma 4 one can easily derive conclusion of the second part of theorem.

Hence the theorem follows. 
Theorem 16. Let $f(z), g(z), k(z)$ and $h(z)$ be any four entire functions such that $0<\lambda_{k}(g) \leq \rho_{k}(g)<\infty$ and $0<\rho_{h}(f)<\infty$. Then

$$
\limsup _{r \rightarrow \infty} \frac{\log M_{h_{n}}^{-1}\left(M_{f_{n, g}}(r)\right)}{\log M_{k}^{-1}\left(M_{g}\left(r^{\delta}\right)\right)} \leq \frac{\rho_{k}(g)}{\lambda_{k}(g)} \text { when } n \text { is even, }
$$

and

$$
\limsup _{r \rightarrow \infty} \frac{\log M_{h_{n}}^{-1}\left(M_{f_{n, g}}(r)\right)}{\log M_{k}^{-1}\left(M_{g}\left(r^{\delta}\right)\right)} \leq \frac{\rho_{h}(f)}{\lambda_{k}(g)} \text { when is any odd integer }
$$

where $\delta>1$.

The proof of Theorem 16 is omitted as it can be carried out in the line of Theorem 15 .

Now we state the following two theorems without their proofs as those can easily be carried out in the line of Theorem 15 and Theorem 16 respectively and with the help of Lemma 4.

Theorem 17. Let $f(z), g(z), k(z)$ and $h(z)$ be any four entire functions such that $0<\lambda_{h}(f) \leq \rho_{h}(f)<\infty$ and $0<\lambda_{k}(g) \leq \rho_{k}(g)<\infty$. Then

$$
\liminf _{r \rightarrow \infty} \frac{\log M_{h_{n}}^{-1}\left(M_{f_{n, g}}(r)\right)}{\log M_{h}^{-1}\left(M_{f}\left(r^{\delta}\right)\right)} \leq \frac{\lambda_{k}(g)}{\lambda_{h}(f)} \text { when } n \text { is even, }
$$

and

$$
\liminf _{r \rightarrow \infty} \frac{\log M_{h_{n}}^{-1}\left(M_{f_{n, g}}(r)\right)}{\log M_{h}^{-1}\left(M_{f}\left(r^{\delta}\right)\right)} \leq 1 \text { when } n \text { is any odd integer }
$$

where $\delta>1$.

Theorem 18. Let $f(z), g(z), k(z)$ and $h(z)$ be any four entire functions such that $0<\lambda_{h}(f) \leq \rho_{h}(f)<\infty$ and $0<\lambda_{k}(g) \leq \rho_{k}(g)<\infty$. Then

$$
\liminf _{r \rightarrow \infty} \frac{\log M_{h_{n}}^{-1}\left(M_{f_{n, g}}(r)\right)}{\log M_{k}^{-1}\left(M_{g}\left(r^{\delta}\right)\right)} \leq 1 \text { when } n \text { is even, }
$$

and

$$
\liminf _{r \rightarrow \infty} \frac{\log M_{h_{n}}^{-1}\left(M_{f_{n, g}}(r)\right)}{\log M_{k}^{-1}\left(M_{g}\left(r^{\delta}\right)\right)} \leq \frac{\lambda_{h}(f)}{\lambda_{k}(g)} \text { when } n \text { is any odd integer }
$$

where $\delta>1$.

Theorem 19. Let $f(z), g(z), k(z)$ and $h(z)$ be any four entire functions such that $0<\lambda_{h}(f)<\infty$ and $0<\lambda_{k}(g)<\infty$. Then for any even number $n$,

$$
\liminf _{r \rightarrow \infty} \frac{\log M_{h_{n}}^{-1}\left(M_{f_{n, g}}(r)\right)}{\log M_{h}^{-1}\left(M_{f}\left(r^{\delta}\right)\right)} \geq \frac{\lambda_{k}(g)}{\rho_{h}(f)} \text { when } 0<\rho_{h}(f)<\infty
$$

and

$$
\liminf _{r \rightarrow \infty} \frac{\log M_{h_{n}}^{-1}\left(M_{f_{n, g}}(r)\right)}{\log M_{k}^{-1}\left(M_{g}\left(r^{\delta}\right)\right)} \geq \frac{\lambda_{k}(g)}{\rho_{k}(g)} \text { when } 0<\rho_{k}(g)<\infty,
$$

where $\delta<1$. 
Proof. From the first part of Lemma 5, we obtain for all sufficiently large values of $r$ that

$$
\log M_{h_{n}}^{-1}\left(M_{f_{n, g}}(r)\right)>\delta\left(\lambda_{k}(g)-\varepsilon\right) \log \left(\frac{r}{18^{n}}\right)+\log \left(\frac{1}{\gamma_{n}}\right),
$$

where $\gamma_{n}$ is defined in Lemma 5 .

Also from the definition of $\rho_{h}(f)$, we obtain for all sufficiently large values of $r$ that

$$
\log M_{h}^{-1}\left(M_{f}\left(r^{\delta}\right)\right) \leq \delta\left(\rho_{h}(f)+\varepsilon\right) \log r .
$$

Analogously,from the definition of $\rho_{k}(g)$, it follows for all sufficiently large values of $r$ that

$$
\log M_{k}^{-1}\left(M_{g}\left(r^{\delta}\right)\right) \leq \delta\left(\rho_{k}(g)+\varepsilon\right) \log r .
$$

Now from (2.21) and (2.22), it follows for all sufficiently large values of $r$ that

$$
\begin{gathered}
\frac{\log M_{h_{n}}^{-1}\left(M_{f_{n, g}}(r)\right)}{\log M_{h}^{-1}\left(M_{f}\left(r^{\delta}\right)\right)}>\frac{\delta\left(\lambda_{k}(g)-\varepsilon\right) \log \left(\frac{r}{18^{n}}\right)+\log \left(\frac{1}{\gamma_{n}}\right)}{\delta\left(\rho_{h}(f)+\varepsilon\right) \log r} \\
\text { i.e., } \liminf _{r \rightarrow \infty} \frac{\log M_{h_{n}}^{-1}\left(M_{f_{n, g}}(r)\right)}{\log M_{h}^{-1}\left(M_{f}\left(r^{\delta}\right)\right)} \geq \frac{\lambda_{k}(g)}{\rho_{h}(f)} .
\end{gathered}
$$

Thus the first part of theorem follows from (2.24).

Similarly, the conclusion of the second part of theorem can easily be derived from (2.21) and (2.23).

Hence the theorem follows.

Theorem 20. Let $f(z), g(z), k(z)$ and $h(z)$ be any four entire functions such that $0<\lambda_{h}(f)<\infty$ and $0<\lambda_{k}(g)<\infty$. Then for any odd number $n$,

$$
\liminf _{r \rightarrow \infty} \frac{\log M_{h_{n}}^{-1}\left(M_{f_{n, g}}(r)\right)}{\log M_{h}^{-1}\left(M_{f}\left(r^{\delta}\right)\right)} \geq \frac{\lambda_{h}(f)}{\rho_{h}(f)} \text { when } 0<\rho_{h}(f)<\infty
$$

and

$$
\liminf _{r \rightarrow \infty} \frac{\log M_{h_{n}}^{-1}\left(M_{f_{n, g}}(r)\right)}{\log M_{k}^{-1}\left(M_{g}\left(r^{\delta}\right)\right)} \geq \frac{\lambda_{h}(f)}{\rho_{k}(g)} \text { when } 0<\rho_{k}(g)<\infty,
$$

where $\delta<1$.

The proof of Theorem 20 is omitted as it can be carried out in the line of Theorem 19 and with the help of the second part of Lemma 5 .

Now we state the following two theorems without their proofs as those can easily be carried out in the line of Theorem 19 and Theorem 20 respectively and with the help of Lemma 5 .

Theorem 21. Let $f(z), g(z), k(z)$ and $h(z)$ be any four entire functions such that $0<\lambda_{h}(f)<\infty$ and $0<\lambda_{k}(g) \leq \rho_{k}(g)<\infty$. Then for any even number $n$,

$$
\limsup _{r \rightarrow \infty} \frac{\log M_{h_{n}}^{-1}\left(M_{f_{n, g}}(r)\right)}{\log M_{h}^{-1}\left(M_{f}\left(r^{\delta}\right)\right)} \geq \frac{\rho_{k}(g)}{\rho_{h}(f)} \text { when } 0<\rho_{h}(f)<\infty
$$


and

$$
\limsup _{r \rightarrow \infty} \frac{\log M_{h_{n}}^{-1}\left(M_{f_{n, g}}(r)\right)}{\log M_{k}^{-1}\left(M_{g}\left(r^{\delta}\right)\right)} \geq 1, \text { when } 0<\rho_{k}(g)<\infty,
$$

where $\delta<1$.

Theorem 22. Let $f(z), g(z), k(z)$ and $h(z)$ be any four entire functions such that $0<\lambda_{h}(f) \leq \rho_{h}(f)<\infty$ and $0<\lambda_{k}(g)<\infty$. Then for any odd number $n$,

$$
\limsup _{r \rightarrow \infty} \frac{\log M_{h_{n}}^{-1}\left(M_{f_{n, g}}(r)\right)}{\log M_{k}^{-1}\left(M_{g}\left(r^{\delta}\right)\right)} \geq \frac{\rho_{h}(f)}{\rho_{k}(g)} \text { when } 0<\rho_{k}(g)<\infty
$$

and

$$
\limsup _{r \rightarrow \infty} \frac{\log M_{h_{n}}^{-1}\left(M_{f_{n, g}}(r)\right)}{\log M_{h}^{-1}\left(M_{f}\left(r^{\delta}\right)\right)} \geq 1 \text { when } 0<\rho_{h}(f)<\infty
$$

where $\delta<1$.

Theorem 23. Let $F(z), G(z), H(z), K(z), f(z), g(z), h(z)$ and $k(z)$ are all entire functions such that $0<\lambda_{H}(F)<\infty, 0<\lambda_{K}(G)<\infty, 0<\rho_{h}(f)<\infty$ and $0<\rho_{k}(g)<\infty$. Then for any two integers $m$ and $n$

$$
\text { (i) } \lim _{r \rightarrow \infty} \frac{M_{H_{m}}^{-1}\left(M_{F_{m, G}}(r)\right)}{M_{h_{n}}^{-1}\left(M_{f_{n, g}}(r)\right) \cdot \log M_{h}^{-1}\left(M_{f}(r)\right)}=\infty
$$

and

$$
\text { (ii) } \lim _{r \rightarrow \infty} \frac{M_{H_{m}}^{-1}\left(M_{F_{m, G}}(r)\right)}{M_{h_{n}}^{-1}\left(M_{f_{n, g}}(r)\right) \cdot \log M_{k}^{-1}\left(M_{g}(r)\right)}=\infty,
$$

when for any $\delta>1$ be such that

$$
\left\{\begin{array}{l}
\delta^{2} \rho_{k}(g)<\lambda_{K}(G) \text { for } m \text { and } n \text { both even } \\
\delta^{2} \rho_{h}(f)<\lambda_{H}(F) \text { for } m \text { and } n \text { both odd } \\
\delta^{2} \rho_{h}(f)<\lambda_{K}(G) \text { for } m \text { even and } n \text { odd } \\
\delta^{2} \rho_{k}(g)<\lambda_{H}(F) \text { for } m \text { odd and } n \text { even } .
\end{array}\right.
$$

Proof. We have from the definition of relative order and for all sufficiently large values of $r$ that

$$
\log M_{h}^{-1}\left(M_{f}(r)\right) \leq\left(\rho_{h}(f)+\varepsilon\right) \log r .
$$

Case I. Let $m$ and $n$ are any two even numbers.

Therefore in view of first part of Lemma 4, we get for all sufficiently large values of $r$ that

$$
M_{h_{n}}^{-1}\left(M_{f_{n, g}}(r)\right)<(r)^{\delta\left(\rho_{k}(g)+\varepsilon\right)},
$$

where $\delta>1$. 
So from (2.26) and (2.27) it follows for all sufficiently large values of $r$ that

$$
M_{h_{n}}^{-1}\left(M_{f_{n, g}}(r)\right) \cdot \log M_{h}^{-1}\left(M_{f}(r)\right)<(r)^{\delta\left(\rho_{k}(g)+\varepsilon\right)} \cdot\left(\rho_{h}(f)+\varepsilon\right) \log r .
$$

Also from first part of Lemma 5, we obtain for all sufficiently large values of $r$ that

$$
M_{H_{m}}^{-1}\left(M_{F_{m, G}}(r)\right)>\left(\frac{1}{\gamma_{m}}\right)^{\frac{1}{\delta}}\left(\frac{r}{18^{m}}\right)^{\frac{\left(\lambda_{K}(G)-\varepsilon\right)}{\delta}},
$$

where $\delta>1$ and $\gamma_{m}$ is defined in Lemma 5 .

Hence combining (2.28) and (2.29) we get for all sufficiently large values of $r$ that,

$$
\frac{M_{H_{m}}^{-1}\left(M_{F_{m, G}}(r)\right)}{M_{h_{n}}^{-1}\left(M_{f_{n, g}}(r)\right) \cdot \log M_{h}^{-1}\left(M_{f}(r)\right)}>\frac{\left(\frac{1}{\gamma_{m}}\right)^{\frac{1}{\delta}}\left(\frac{r}{18^{m}}\right)^{\frac{\left(\lambda_{K}(G)-\varepsilon\right)}{\delta}}}{(r)^{\delta\left(\rho_{k}(g)+\varepsilon\right)} \cdot\left(\rho_{h}(f)+\varepsilon\right) \log r} .
$$

Since $\delta^{2} \rho_{k}(g)<\lambda_{K}(G)$, we can choose $\varepsilon(>0)$ in such a manner that

$$
\delta^{2}\left(\rho_{k}(g)+\varepsilon\right) \leq\left(\lambda_{K}(G)-\varepsilon\right) .
$$

Thus from (2.30) and (2.31) we obtain that

$$
\lim _{r \rightarrow \infty} \frac{M_{H_{m}}^{-1}\left(M_{F_{m, G}}(r)\right)}{M_{h_{n}}^{-1}\left(M_{f_{n, g}}(r)\right) \cdot \log M_{h}^{-1}\left(M_{f}(r)\right)}=\infty .
$$

Case II. Let $m$ and $n$ are any two odd numbers .

Now in view of second part of Lemma 4, we get for all sufficiently large values of $r$ that

$$
M_{h_{n}}^{-1}\left(M_{f_{n, g}}(r)\right)<(r)^{\delta\left(\rho_{h}(f)+\varepsilon\right)},
$$

where $\delta>1$.

So from (2.26) and (2.33) it follows for all sufficiently large values of $r$ that

$$
M_{h_{n}}^{-1}\left(M_{f_{n, g}}(r)\right) \cdot \log M_{h}^{-1}\left(M_{f}(r)\right)<(r)^{\delta\left(\rho_{h}(f)+\varepsilon\right)} \cdot\left(\rho_{h}(f)+\varepsilon\right) \log r .
$$
that

Also from second part of Lemma 5 , we obtain for all sufficiently large values of $r$

$$
M_{H_{m}}^{-1}\left(M_{F_{m, G}}(r)\right)>\left(\frac{1}{\gamma_{m}}\right)^{\frac{1}{\delta}}\left(\frac{r}{18^{m}}\right)^{\frac{\left(\lambda_{H}(F)-\varepsilon\right)}{\delta}} .
$$

Hence combining (2.34) and (2.35) we get for all sufficiently large values of $r$ that,

$$
\frac{M_{H_{m}}^{-1}\left(M_{F_{m, G}}(r)\right)}{M_{h_{n}}^{-1}\left(M_{f_{n, g}}(r)\right) \cdot \log M_{h}^{-1}\left(M_{f}(r)\right)}>\frac{\left(\frac{1}{\gamma_{m}}\right)^{\frac{1}{\delta}}\left(\frac{r}{18^{m}}\right)^{\frac{\left(\lambda_{H}(F)-\varepsilon\right)}{\delta}}}{(r)^{\delta\left(\rho_{h}(f)+\varepsilon\right)} \cdot\left(\rho_{h}(f)+\varepsilon\right) \log r} .
$$

As $\delta^{2} \rho_{h}(f)<\lambda_{H}(F)$, we can choose $\varepsilon(>0)$ in such a manner that

$$
\delta^{2}\left(\rho_{h}(f)+\varepsilon\right) \leq\left(\lambda_{H}(F)-\varepsilon\right) .
$$


Therefore from (2.36) and (2.37) it follows that

$$
\lim _{r \rightarrow \infty} \frac{M_{H_{m}}^{-1}\left(M_{F_{m, G}}(r)\right)}{M_{h_{n}}^{-1}\left(M_{f_{n, g}}(r)\right) \cdot \log M_{h}^{-1}\left(M_{f}(r)\right)}=\infty .
$$

Case III. Let $m$ be any even number and $n$ be any odd number.

Then combining (2.29) and (2.34) we get for all sufficiently large values of $r$ that

$$
\frac{M_{H_{m}}^{-1}\left(M_{F_{m, G}}(r)\right)}{M_{h_{n}}^{-1}\left(M_{f_{n, g}}(r)\right) \cdot \log M_{h}^{-1}\left(M_{f}(r)\right)}>\frac{\left(\frac{1}{\gamma_{m}}\right)^{\frac{1}{\delta}}\left(\frac{r}{18^{m}}\right)^{\frac{\left(\lambda_{K}(G)-\varepsilon\right)}{\delta}}}{(r)^{\delta\left(\rho_{h}(f)+\varepsilon\right)} \cdot\left(\rho_{h}(f)+\varepsilon\right) \log r} .
$$

Since $\delta^{2} \rho_{h}(f)<\lambda_{K}(G)$, we can choose $\varepsilon(>0)$ in such a manner that

$$
\delta^{2}\left(\rho_{h}(f)+\varepsilon\right) \leq\left(\lambda_{K}(G)-\varepsilon\right) .
$$

So from (2.39) and (2.40) we get that

$$
\lim _{r \rightarrow \infty} \frac{M_{H_{m}}^{-1}\left(M_{F_{m, G}}(r)\right)}{M_{h_{n}}^{-1}\left(M_{f_{n, g}}(r)\right) \cdot \log M_{h}^{-1}\left(M_{f}(r)\right)}=\infty .
$$

Case IV. Let $m$ be any odd number and $n$ be any even number .

Therefore combining (2.28) and (2.35) we obtain for all sufficiently large values of $r$ that

$$
\frac{M_{H_{m}}^{-1}\left(M_{F_{m, G}}(r)\right)}{M_{h_{n}}^{-1}\left(M_{f_{n, g}}(r)\right) \cdot \log M_{h}^{-1}\left(M_{f}(r)\right)}>\frac{\left(\frac{1}{\gamma_{m}}\right)^{\frac{1}{\delta}}\left(\frac{r}{18^{m}}\right)^{\frac{\left(\lambda_{H}(F)-\varepsilon\right)}{\delta}}}{(r)^{\delta\left(\rho_{k}(g)+\varepsilon\right)} \cdot\left(\rho_{h}(f)+\varepsilon\right) \log r} .
$$

As $\delta^{2} \rho_{k}(g)<\lambda_{H}(F)$, we can choose $\varepsilon(>0)$ in such a manner that

$$
\delta^{2}\left(\rho_{k}(g)+\varepsilon\right) \leq\left(\lambda_{H}(F)-\varepsilon\right) .
$$

Hence from (2.42) and (2.43) we have

$$
\lim _{r \rightarrow \infty} \frac{M_{H_{m}}^{-1}\left(M_{F_{m, G}}(r)\right)}{M_{h_{n}}^{-1}\left(M_{f_{n, g}}(r)\right) \cdot \log M_{h}^{-1}\left(M_{f}(r)\right)}=\infty .
$$

Thus the first part of the theorem follows from (2.32), (2.38), (2.41) and (2.44). Similarly, from the definition of $\rho_{k}(g)$ one can easily derive the conclusion of the second part of the theorem.

Hence the theorem follows.

Remark 9. If we consider $\rho_{K}(G), \rho_{H}(F), \rho_{K}(G)$ and $\rho_{H}(F)$ instead of $\lambda_{K}(G)$, $\lambda_{H}(F), \lambda_{K}(G)$ and $\lambda_{H}(F)$ respectively in (2.25) and the other conditions remain the same, the conclusion of Theorem 23 is remain valid with "limit superior" replaced by "limit". 
Theorem 24. Let $f(z), g(z), k(z)$ and $h(z)$ be any four entire functions such that $0<\rho_{h}(f)<\infty, 0<\rho_{k}(g)<\infty$ and $\sigma_{k}(g)<\infty$. Then for any even number $n$,

$$
\limsup _{r \rightarrow \infty} \frac{M_{h_{n}}^{-1}\left(M_{f_{n, g}}(r)\right)}{\log M_{h}^{-1}\left(M_{f}\left(\exp (r)^{\delta \rho_{k}(g)}\right)\right)} \leq \frac{\left(\sigma_{k}(g)\right)^{\delta}}{\lambda_{h}(f)} \text { if } \lambda_{h}(f) \neq 0
$$

and

$$
\limsup _{r \rightarrow \infty} \frac{M_{h_{n}}^{-1}\left(M_{f_{n, g}}(r)\right)}{\log M_{k}^{-1}\left(M_{g}\left(\exp (r)^{\delta \rho_{k}(g)}\right)\right)} \leq \frac{\left(\sigma_{k}(g)\right)^{\delta}}{\lambda_{k}(g)} \quad \text { if } \lambda_{k}(g) \neq 0,
$$

where $\delta>1$.

Proof. In view of the first part of Lemma 4 we have for all sufficiently large values of $r$ that

$$
\begin{aligned}
& \frac{M_{h_{n}}^{-1}\left(M_{f_{n, g}}(r)\right)}{\log M_{h}^{-1}\left(M_{f}\left(\exp (r)^{\delta \rho_{k}(g)}\right)\right)}<\frac{\left(M_{k}^{-1}\left(M_{g}(r)\right)\right)^{\delta}}{\log M_{h}^{-1}\left(M_{f}\left(\exp (r)^{\delta \rho_{k}(g)}\right)\right)} \\
& \frac{M_{h_{n}}^{-1}\left(M_{f_{n, g}}(r)\right)}{\log M_{h}^{-1}\left(M_{f}\left(\exp (r)^{\delta \rho_{k}(g)}\right)\right)}<\left(\frac{M_{k}^{-1}\left(M_{g}(r)\right)}{r^{\rho_{k}(g)}}\right) \cdot \frac{\log \exp (r)^{\delta \rho_{k}(g)}}{\log M_{h}^{-1}\left(M_{f}\left(\exp (r)^{\delta \rho_{k}(g)}\right)\right)} \\
& \quad \text { i.e., } \quad \limsup _{r \rightarrow \infty} \frac{M_{h_{n}}^{-1}\left(M_{f_{n, g}}(r)\right)}{\log M_{h}^{-1}\left(M_{f}\left(\exp (r)^{\delta \rho_{k}(g)}\right)\right)} \\
& \quad \leq\left(\limsup _{r \rightarrow \infty} \frac{M_{k}^{-1}\left(M_{g}(r)\right)}{r^{\rho_{k}(g)}}\right)^{\delta} \cdot \limsup _{r \rightarrow \infty} \frac{\log \exp (r)^{\delta \rho_{k}(g)}}{\log M_{h}^{-1}\left(M_{f}\left(\exp (r)^{\delta \rho_{k}(g)}\right)\right)} \\
& \text { i.e., } \limsup _{r \rightarrow \infty} \frac{M_{h_{n}}^{-1}\left(M_{f_{n, g}}(r)\right)}{\log M_{h}^{-1}\left(M_{f}\left(\exp (r)^{\delta \rho_{k}(g)}\right)\right)} \leq\left(\sigma_{k}(g)\right)^{\delta} \cdot \frac{1}{\lambda_{h}(f)}=\frac{\left(\sigma_{k}(g)\right)^{\delta}}{\lambda_{h}(f)} .
\end{aligned}
$$

Thus the first part of theorem is established.

Similarly, with the help of the first part of Lemma 4 one can easily derive conclusion of the second part of theorem.

Hence the theorem follows.

Theorem 25. Let $f(z), g(z), k(z)$ and $h(z)$ be any four entire functions such that $0<\lambda_{h}(f) \leq \rho_{h}(f)<\infty, 0<\lambda_{k}(g) \leq \rho_{k}(g)<\infty$ and $\sigma_{k}(g)<\infty$. Then for any even number $n$,

$$
\liminf _{r \rightarrow \infty} \frac{M_{h_{n}}^{-1}\left(M_{f_{n, g}}(r)\right)}{\log M_{h}^{-1}\left(M_{f}\left(\exp (r)^{\delta \rho_{k}(g)}\right)\right)} \leq \min \left\{\frac{\left(\bar{\sigma}_{k}(g)\right)^{\delta}}{\lambda_{h}(f)}, \frac{\left(\sigma_{k}(g)\right)^{\delta}}{\rho_{h}(f)}\right\}
$$


and

$$
\liminf _{r \rightarrow \infty} \frac{M_{h_{n}}^{-1}\left(M_{f_{n, g}}(r)\right)}{\log M_{k}^{-1}\left(M_{g}\left(\exp (r)^{\delta \rho_{k}(g)}\right)\right)} \leq \min \left\{\frac{\left(\bar{\sigma}_{k}(g)\right)^{\delta}}{\lambda_{k}(g)}, \frac{\left(\sigma_{k}(g)\right)^{\delta}}{\rho_{k}(g)}\right\}
$$

where $\delta>1$.

Proof of Theorem 25 is omitted as it can be carried out in the line of Theorem 24 and with help of the first part of Lemma 4.

Now we state the following two theorems without their proofs as those can easily be carried out with the help of second part of Lemma 4 and in the line of Theorem 24 and Theorem 25 respectively.

Theorem 26. Let $f(z), g(z), k(z)$ and $h(z)$ be any four entire functions such that $0<\rho_{h}(f)<\infty, 0<\rho_{k}(g)<\infty$ and $\sigma_{h}(f)<\infty$. Then for any odd number $n$,

$$
\limsup _{r \rightarrow \infty} \frac{M_{h_{n}}^{-1}\left(M_{f_{n, g}}(r)\right)}{\log M_{h}^{-1}\left(M_{f}\left(\exp (r)^{\delta \rho_{h}(f)}\right)\right)} \leq \frac{\left(\sigma_{h}(f)\right)^{\delta}}{\lambda_{h}(f)} \text { if } \lambda_{h}(f) \neq 0
$$

and

$$
\limsup _{r \rightarrow \infty} \frac{M_{h_{n}}^{-1}\left(M_{f_{n, g}}(r)\right)}{\log M_{k}^{-1}\left(M_{g}\left(\exp (r)^{\delta \rho_{h}(f)}\right)\right)} \leq \frac{\left(\sigma_{h}(f)\right)^{\delta}}{\lambda_{k}(g)} \quad \text { if } \lambda_{k}(g) \neq 0
$$

where $\delta>1$.

Theorem 27. Let $f(z), g(z), k(z)$ and $h(z)$ be any four entire functions such that $0<\lambda_{h}(f) \leq \rho_{h}(f)<\infty, 0<\lambda_{k}(g) \leq \rho_{k}(g)<\infty$ and $\sigma_{h}(f)<\infty$. Then for any odd number $n$,

$$
\liminf _{r \rightarrow \infty} \frac{M_{h_{n}}^{-1}\left(M_{f_{n, g}}(r)\right)}{\log M_{h}^{-1}\left(M_{f}\left(\exp (r)^{\delta \rho_{h}(f)}\right)\right)} \leq \min \left\{\frac{\left(\bar{\sigma}_{h}(f)\right)^{\delta}}{\lambda_{h}(f)}, \frac{\left(\sigma_{h}(f)\right)^{\delta}}{\rho_{h}(f)}\right\}
$$

and

$$
\liminf _{r \rightarrow \infty} \frac{M_{h_{n}}^{-1}\left(M_{f_{n, g}}(r)\right)}{\log M_{k}^{-1}\left(M_{g}\left(\exp (r)^{\delta \rho_{h}(f)}\right)\right)} \leq \min \left\{\frac{\left(\bar{\sigma}_{h}(f)\right)^{\delta}}{\lambda_{k}(g)}, \frac{\left(\sigma_{h}(f)\right)^{\delta}}{\rho_{k}(g)}\right\}
$$

where $\delta>1$.

Analogously, one may state the following four theorems without their proofs on the basis of relative weak type of entire function with respect to another entire function :

Theorem 28. Let $f(z), g(z), k(z)$ and $h(z)$ be any four entire functions such that $0<\rho_{h}(f)<\infty, 0<\rho_{k}(g)<\infty$ and $\bar{\tau}_{k}(g)<\infty$. Then for any even number $n$,

$$
\limsup _{r \rightarrow \infty} \frac{M_{h_{n}}^{-1}\left(M_{f_{n, g}}(r)\right)}{\log M_{h}^{-1}\left(M_{f}\left(\exp (r)^{\delta \lambda_{k}(g)}\right)\right)} \leq \frac{\left(\bar{\tau}_{k}(g)\right)^{\delta}}{\lambda_{h}(f)} \text { if } \lambda_{h}(f) \neq 0
$$


and

$$
\limsup _{r \rightarrow \infty} \frac{M_{h_{n}}^{-1}\left(M_{f_{n, g}}(r)\right)}{\log M_{k}^{-1}\left(M_{g}\left(\exp (r)^{\delta \lambda_{k}(g)}\right)\right)} \leq \frac{\left(\bar{\tau}_{k}(g)\right)^{\delta}}{\lambda_{k}(g)} \quad \text { if } \lambda_{k}(g) \neq 0,
$$

where $\delta>1$.

Theorem 29. Let $f(z), g(z), k(z)$ and $h(z)$ be any four entire functions such that $0<\lambda_{h}(f) \leq \rho_{h}(f)<\infty, 0<\lambda_{k}(g) \leq \rho_{k}(g)<\infty$ and $\bar{\tau}_{k}(g)<\infty$. Then for any even number $n$,

$$
\liminf _{r \rightarrow \infty} \frac{M_{h_{n}}^{-1}\left(M_{f_{n, g}}(r)\right)}{\log M_{h}^{-1}\left(M_{f}\left(\exp (r)^{\delta \lambda_{k}(g)}\right)\right)} \leq \min \left\{\frac{\left(\tau_{k}(g)\right)^{\delta}}{\lambda_{h}(f)}, \frac{\left(\bar{\tau}_{k}(g)\right)^{\delta}}{\rho_{h}(f)}\right\}
$$

and

$$
\liminf _{r \rightarrow \infty} \frac{M_{h_{n}}^{-1}\left(M_{f_{n, g}}(r)\right)}{\log M_{k}^{-1}\left(M_{g}\left(\exp (r)^{\delta \lambda_{k}(g)}\right)\right)} \leq \min \left\{\frac{\left(\tau_{k}(g)\right)^{\delta}}{\lambda_{k}(g)}, \frac{\left(\bar{\tau}_{k}(g)\right)^{\delta}}{\rho_{k}(g)}\right\}
$$

where $\delta>1$.

Theorem 30. Let $f(z), g(z), k(z)$ and $h(z)$ be any four entire functions such that $0<\rho_{h}(f)<\infty, 0<\rho_{k}(g)<\infty$ and $\bar{\tau}_{h}(f)<\infty$. Then for any odd number $n$,

$$
\limsup _{r \rightarrow \infty} \frac{M_{h_{n}}^{-1}\left(M_{f_{n, g}}(r)\right)}{\log M_{h}^{-1}\left(M_{f}\left(\exp (r)^{\delta \lambda_{h}(f)}\right)\right)} \leq \frac{\left(\bar{\tau}_{h}(f)\right)^{\delta}}{\lambda_{h}(f)} \text { if } \lambda_{h}(f) \neq 0
$$

and

$$
\limsup _{r \rightarrow \infty} \frac{M_{h_{n}}^{-1}\left(M_{f_{n, g}}(r)\right)}{\log M_{k}^{-1}\left(M_{g}\left(\exp (r)^{\delta \lambda_{h}(f)}\right)\right)} \leq \frac{\left(\bar{\tau}_{h}(f)\right)^{\delta}}{\lambda_{k}(g)} \quad \text { if } \lambda_{k}(g) \neq 0
$$

where $\delta>1$.

Theorem 31. Let $f(z), g(z), k(z)$ and $h(z)$ be any four entire functions such that $0<\lambda_{h}(f) \leq \rho_{h}(f)<\infty, 0<\lambda_{k}(g) \leq \rho_{k}(g)<\infty$ and $\bar{\tau}_{h}(f)<\infty$. Then for any odd number $n$,

$$
\liminf _{r \rightarrow \infty} \frac{M_{h_{n}}^{-1}\left(M_{f_{n, g}}(r)\right)}{\log M_{h}^{-1}\left(M_{f}\left(\exp (r)^{\delta \lambda_{h}(f)}\right)\right)} \leq \min \left\{\frac{\left(\tau_{h}(f)\right)^{\delta}}{\lambda_{h}(f)}, \frac{\left(\bar{\tau}_{h}(f)\right)^{\delta}}{\rho_{h}(f)}\right\}
$$

and

$$
\liminf _{r \rightarrow \infty} \frac{M_{h_{n}}^{-1}\left(M_{f_{n, g}}(r)\right)}{\log M_{k}^{-1}\left(M_{g}\left(\exp (r)^{\delta \lambda_{h}(f)}\right)\right)} \leq \min \left\{\frac{\left(\tau_{h}(f)\right)^{\delta}}{\lambda_{k}(g)}, \frac{\left(\bar{\tau}_{h}(f)\right)^{\delta}}{\rho_{k}(g)}\right\}
$$

where $\delta>1$. 
Theorem 32. Let $f(z), g(z), k(z)$ and $h(z)$ be any four entire functions such that $0<\lambda_{h}(f)<\infty, 0<\lambda_{k}(g)<\infty$ and $\bar{\sigma}_{k}(g)>0$. Then for any even number $n$ and $\delta<1$

$$
\liminf _{r \rightarrow \infty} \frac{M_{h_{n}}^{-1}\left(M_{f_{n, g}}(r)\right)}{\log M_{h}^{-1}\left(M_{f}\left(\exp (r)^{\delta \rho_{k}(g)}\right)\right)} \geq A \frac{\bar{\sigma}_{k}(g)}{\rho_{h}(f)} \text { if } \rho_{h}(f)<\infty
$$

and

$$
\liminf _{r \rightarrow \infty} \frac{M_{h_{n}}^{-1}\left(M_{f_{n, g}}(r)\right)}{\log M_{k}^{-1}\left(M_{g}\left(\exp (r)^{\delta \rho_{k}(g)}\right)\right)} \geq A \frac{\bar{\sigma}_{k}(g)}{\rho_{k}(g)} \text { if } \rho_{k}(g)<\infty,
$$

where $A=\frac{1}{\left[18^{n \rho_{k}(g)} \cdot \gamma_{n}\right]^{\delta}}$ and $\gamma_{n}$ is defined in Lemma 5 .

Proof. From the first part of Lemma 5, we obtain for all sufficiently large values of $r$ that

$$
\begin{gathered}
M_{h_{n}}^{-1}\left(M_{f_{n, g}}(r)\right)>\frac{1}{\left[18^{n \rho_{k}}(g) \cdot \gamma_{n}\right]^{\delta}}\left(\bar{\sigma}_{k}(g)-\varepsilon\right) r^{\delta \rho_{k}(g)} \\
\text { i.e., } M_{h_{n}}^{-1}\left(M_{f_{n, g}}(r)\right)>A\left(\bar{\sigma}_{k}(g)-\varepsilon\right) r^{\delta \rho_{k}(g)} .
\end{gathered}
$$

Also from the definition of $\rho_{h}(f)$, we obtain for all sufficiently large values of $r$ that

$$
\log M_{h}^{-1}\left(M_{f}\left(\exp (r)^{\delta \rho_{k}(g)}\right)\right) \leq\left(\rho_{h}(f)+\varepsilon\right) r^{\delta \rho_{k}(g)} .
$$

Analogously,from the definition of $\rho_{k}(g)$, it follows for all sufficiently large values of $r$ that

$$
\log M_{k}^{-1}\left(M_{g}\left(\exp (r)^{\delta \rho_{k}(g)}\right)\right) \leq\left(\rho_{k}(g)+\varepsilon\right) r^{\delta \rho_{k}(g)} .
$$

Now from (2.45) and (2.46), it follows for all sufficiently large values of $r$ that

$$
\begin{aligned}
& \frac{M_{h_{n}}^{-1}\left(M_{f_{n, g}}(r)\right)}{\log M_{h}^{-1}\left(M_{f}\left(\exp (r)^{\delta \rho_{k}(g)}\right)\right)}>A \frac{\left(\bar{\sigma}_{k}(g)-\varepsilon\right) r^{\delta \rho_{k}(g)}}{\left(\rho_{h}(f)+\varepsilon\right) r^{\delta \rho_{k}(g)}} \\
& \text { i.e., } \liminf _{r \rightarrow \infty} \frac{M_{h_{n}}^{-1}\left(M_{f_{n, g}}(r)\right)}{\log M_{h}^{-1}\left(M_{f}\left(\exp (r)^{\delta \rho_{k}(g)}\right)\right)} \geq A \frac{\bar{\sigma}_{k}(g)}{\rho_{h}(f)} .
\end{aligned}
$$

Thus the first part of theorem follows from (2.48).

Like wise, the conclusion of the second part of theorem can easily be derived from (2.45) and (2.47) .

Hence the theorem follows.

Theorem 33. Let $f(z), g(z), k(z)$ and $h(z)$ be any four entire functions such that $0<\lambda_{h}(f) \leq \rho_{h}(f)<\infty, 0<\lambda_{k}(g) \leq \rho_{k}(g)<\infty$ and $\bar{\sigma}_{k}(g)>0$. Then for any even number $n$ and $\delta<1$

$$
\limsup _{r \rightarrow \infty} \frac{M_{h_{n}}^{-1}\left(M_{f_{n, g}}(r)\right)}{\log M_{h}^{-1}\left(M_{f}\left(\exp (r)^{\delta \rho_{k}(g)}\right)\right)} \geq A \cdot \max \left\{\frac{\sigma_{k}(g)}{\rho_{h}(f)}, \frac{\bar{\sigma}_{k}(g)}{\lambda_{h}(f)}\right\}
$$


and

$$
\limsup _{r \rightarrow \infty} \frac{M_{h_{n}}^{-1}\left(M_{f_{n, g}}(r)\right)}{\log M_{k}^{-1}\left(M_{g}\left(\exp (r)^{\delta \rho_{k}(g)}\right)\right)} \geq A \cdot \max \left\{\frac{\sigma_{k}(g)}{\rho_{k}(g)}, \frac{\bar{\sigma}_{k}(g)}{\lambda_{k}(g)}\right\},
$$

where $A=\frac{1}{\left[18^{n \rho_{k}(g)} \cdot \gamma_{n}\right]^{\delta}}$ and $\gamma_{n}$ is defined in Lemma 5 .

Proof of Theorem 33 is omitted as it can be carried out in the line of Theorem 32 and with help of the first part of Lemma 5.

Similarly, we state the following two theorems without their proofs as those can easily be carried out with the help of second part of Lemma 5 and in the line of Theorem 32 and Theorem 33 respectively.

Theorem 34. Let $f(z), g(z), k(z)$ and $h(z)$ be any four entire functions such that $0<\lambda_{h}(f)<\infty, 0<\lambda_{k}(g)<\infty$ and $\bar{\sigma}_{h}(f)>0$. Then for any odd number $n$ and $\delta<1$

$$
\liminf _{r \rightarrow \infty} \frac{M_{h_{n}}^{-1}\left(M_{f_{n, g}}(r)\right)}{\log M_{h}^{-1}\left(M_{f}\left(\exp (r)^{\delta \rho_{h}(f)}\right)\right)} \geq A \frac{\bar{\sigma}_{h}(f)}{\rho_{h}(f)} \text { if } \rho_{h}(f)<\infty
$$

and

$$
\liminf _{r \rightarrow \infty} \frac{M_{h_{n}}^{-1}\left(M_{f_{n, g}}(r)\right)}{\log M_{k}^{-1}\left(M_{g}\left(\exp (r)^{\delta \rho_{h}(f)}\right)\right)} \geq A \frac{\bar{\sigma}_{h}(f)}{\rho_{k}(g)} \text { if } \rho_{k}(g)<\infty,
$$

where $A=\frac{1}{\left[18^{n \rho_{h}(f)} \cdot \gamma_{n}\right]^{\delta}}$ and $\gamma_{n}$ is defined in Lemma 5 .

Theorem 35. Let $f(z), g(z), k(z)$ and $h(z)$ be any four entire functions such that $0<\lambda_{h}(f) \leq \rho_{h}(f)<\infty, 0<\lambda_{k}(g) \leq \rho_{k}(g)<\infty$ and $\bar{\sigma}_{h}(f)>0$. Then for any odd number $n$ and $\delta<1$

$$
\limsup _{r \rightarrow \infty} \frac{M_{h_{n}}^{-1}\left(M_{f_{n, g}}(r)\right)}{\log M_{h}^{-1}\left(M_{f}\left(\exp (r)^{\delta \rho_{h}(f)}\right)\right)} \geq A \cdot \max \left\{\frac{\sigma_{h}(f)}{\rho_{h}(f)}, \frac{\bar{\sigma}_{h}(f)}{\lambda_{h}(f)}\right\}
$$

and

$$
\limsup _{r \rightarrow \infty} \frac{M_{h_{n}}^{-1}\left(M_{f_{n, g}}(r)\right)}{\log M_{k}^{-1}\left(M_{g}\left(\exp (r)^{\delta \rho_{h}(f)}\right)\right)} \geq A \cdot \max \left\{\frac{\sigma_{h}(f)}{\rho_{k}(g)}, \frac{\bar{\sigma}_{h}(f)}{\lambda_{k}(g)}\right\},
$$

where $A=\frac{1}{\left[18^{n \rho_{h}(f)} \cdot \gamma_{n}\right]^{\delta}}$ and $\gamma_{n}$ is defined in Lemma 5 .

Similarly, one may state the following four theorems without their proofs on the basis of relative weak type of entire function with respect to another entire function:

Theorem 36. Let $f(z), g(z), k(z)$ and $h(z)$ be any four entire functions such that $0<\lambda_{h}(f)<\infty, 0<\lambda_{k}(g)<\infty$ and $\tau_{k}(g)>0$. Then for any even number $n$ and $\delta<1$

$$
\liminf _{r \rightarrow \infty} \frac{M_{h_{n}}^{-1}\left(M_{f_{n, g}}(r)\right)}{\log M_{h}^{-1}\left(M_{f}\left(\exp (r)^{\delta \lambda_{k}(g)}\right)\right)} \geq A \frac{\tau_{k}(g)}{\rho_{h}(f)} \text { if } \rho_{h}(f)<\infty
$$


and

$$
\liminf _{r \rightarrow \infty} \frac{M_{h_{n}}^{-1}\left(M_{f_{n, g}}(r)\right)}{\log M_{k}^{-1}\left(M_{g}\left(\exp (r)^{\delta \lambda_{k}(g)}\right)\right)} \geq A \frac{\tau_{k}(g)}{\rho_{k}(g)} \text { if } \rho_{k}(g)<\infty,
$$

where $A=\frac{1}{\left[18^{n \lambda_{k}(g)} \cdot \gamma_{n}\right]^{\delta}}$ and $\gamma_{n}$ is defined in Lemma 5 .

Theorem 37. Let $f(z), g(z), k(z)$ and $h(z)$ be any four entire functions such that $0<\lambda_{h}(f) \leq \rho_{h}(f)<\infty, 0<\lambda_{k}(g) \leq \rho_{k}(g)<\infty$ and $\tau_{k}(g)>0$. Then for any even number $n$ and $\delta<1$

$$
\limsup _{r \rightarrow \infty} \frac{M_{h_{n}}^{-1}\left(M_{f_{n, g}}(r)\right)}{\log M_{h}^{-1}\left(M_{f}\left(\exp (r)^{\delta \lambda_{k}(g)}\right)\right)} \geq A \cdot \max \left\{\frac{\bar{\tau}_{k}(g)}{\rho_{h}(f)}, \frac{\tau_{k}(g)}{\lambda_{h}(f)}\right\}
$$

and

$$
\limsup _{r \rightarrow \infty} \frac{M_{h_{n}}^{-1}\left(M_{f_{n, g}}(r)\right)}{\log M_{k}^{-1}\left(M_{g}\left(\exp (r)^{\delta \lambda_{k}(g)}\right)\right)} \geq A \cdot \max \left\{\frac{\bar{\tau}_{k}(g)}{\rho_{k}(g)}, \frac{\tau_{k}(g)}{\lambda_{k}(g)}\right\}
$$

where $A=\frac{1}{\left[18^{n \lambda_{k}(g)} \cdot \gamma_{n}\right]^{\delta}}$ and $\gamma_{n}$ is defined in Lemma 5 .

Theorem 38. Let $f(z), g(z), k(z)$ and $h(z)$ be any four entire functions such that $0<\lambda_{h}(f)<\infty, 0<\lambda_{k}(g)<\infty$ and $\tau_{h}(f)>0$. Then for any odd number $n$ and $\delta<1$

$$
\liminf _{r \rightarrow \infty} \frac{M_{h_{n}}^{-1}\left(M_{f_{n, g}}(r)\right)}{\log M_{h}^{-1}\left(M_{f}\left(\exp (r)^{\delta \lambda_{h}(f)}\right)\right)} \geq A \frac{\tau_{h}(f)}{\rho_{h}(f)} \text { if } \rho_{h}(f)<\infty
$$

and

$$
\liminf _{r \rightarrow \infty} \frac{M_{h_{n}}^{-1}\left(M_{f_{n, g}}(r)\right)}{\log M_{k}^{-1}\left(M_{g}\left(\exp (r)^{\delta \lambda_{h}(f)}\right)\right)} \geq A \frac{\tau_{h}(f)}{\rho_{k}(g)} \text { if } \rho_{k}(g)<\infty,
$$

where $A=\frac{1}{\left[18^{n \lambda_{h}(f)} \cdot \gamma_{n}\right]^{\delta}}$ and $\gamma_{n}$ is defined in Lemma 5 .

Theorem 39. Let $f(z), g(z), k(z)$ and $h(z)$ be any four entire functions such that $0<\lambda_{h}(f) \leq \rho_{h}(f)<\infty, 0<\lambda_{k}(g) \leq \rho_{k}(g)<\infty$ and $\tau_{h}(f)>0$. Then for any odd number $n$ and $\delta<1$

$$
\limsup _{r \rightarrow \infty} \frac{M_{h_{n}}^{-1}\left(M_{f_{n, g}}(r)\right)}{\log M_{h}^{-1}\left(M_{f}\left(\exp (r)^{\delta \lambda_{h}(f)}\right)\right)} \geq A \cdot \max \left\{\frac{\bar{\tau}_{h}(f)}{\rho_{h}(f)}, \frac{\tau_{h}(f)}{\lambda_{h}(f)}\right\}
$$

and

$$
\limsup _{r \rightarrow \infty} \frac{M_{h_{n}}^{-1}\left(M_{f_{n, g}}(r)\right)}{\log M_{k}^{-1}\left(M_{g}\left(\exp (r)^{\delta \lambda_{h}(f)}\right)\right)} \geq A \cdot \max \left\{\frac{\bar{\tau}_{h}(f)}{\rho_{k}(g)}, \frac{\tau_{h}(f)}{\lambda_{k}(g)}\right\},
$$

where $A=\frac{1}{\left[18^{n \lambda_{h}(f)} \cdot \gamma_{n}\right]^{\delta}}$ and $\gamma_{n}$ is defined in Lemma 5 . 


\section{Acknowledgment}

The author is thankful to the referee for his/her valuable suggestions towards the improvement of the paper.

\section{References}

[1] D. Banerjee, N. Mondal, Maximum modulus and maximum term of generalized iterated entire functions, Bull. Allahabad Math. Soc. 27 (1) (2012) 117-131.

[2] L. Bernal, Crecimiento relativo de funciones enteras. Contribución al estudio de lasfunciones enteras con indice exponencial finito, Doctoral Dissertation, University of Seville, Spain, 1984.

[3] L. Bernal, Orden relativo de crecimiento de funciones enteras, Collect. Math. 39 (1988) 209-229.

[4] J. Clunie, The composition of entire and meromorphic functions, Mathematical essays dedicated to A.J.Macintyre, Ohio University Press (1970) 75-92.

[5] A.S.B. Holland, Introduction to the Theory of Entire Functions, Academic Press, New York and London, 1973.

[6] B.K. Lahiri, D. Banerjee, Generalized relative order of entire functions, Proc. Nat. Acad. Sci. India 72(A) IV (2002) 351-371.

[7] C. Roy, Some properties of entire functions in one and several complex variables, Ph.D. Thesis, University of Calcutta, 2010.

[8] A.P. Singh, Growth of composite entire functions, Kodai Math. J. 8 (1985) 99102.

[9] E.C. Titchmarsh, The Theory of Functions, 2nd edition, Oxford University Press, Oxford, 1939.

[10] G. Valiron, Lectures on the General Theory of Integral Functions, Chelsea Publishing Company, 1949.

\section{DOI: $10.7862 / \mathrm{rf.2020.2}$}

\section{Tanmay Biswas}

email: tanmaybiswas_math@rediffmail.com

ORCID: 0000-0001-6984-6897

Rajbari, Rabindrapalli, R.N. Tagore Road,

P.O.- Krishnagar, Dist-Nadia, PIN- 741101 
West Bengal

INDIA

Received 21.11.2019

Accepted 04.05.2020 